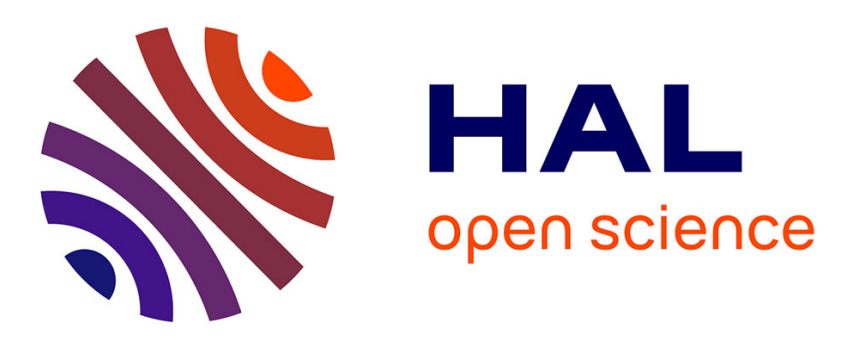

\title{
Influence of viscoelastic and viscous absorption on ultrasonic wave propagation in cortical bone: Application to axial transmission
}

S. Naili, M.B. Vu, Q. Grimal, M. Talmant, Christophe Desceliers, Christian Soize, G. Haïat

\section{To cite this version:}

S. Naili, M.B. Vu, Q. Grimal, M. Talmant, Christophe Desceliers, et al.. Influence of viscoelastic and viscous absorption on ultrasonic wave propagation in cortical bone: Application to axial transmission. Journal of the Acoustical Society of America, 2010, 127 (4), pp.2622-2634. 10.1121/1.3353091 . hal00684320

\section{HAL Id: hal-00684320 \\ https://hal.science/hal-00684320}

Submitted on 1 Apr 2012

HAL is a multi-disciplinary open access archive for the deposit and dissemination of scientific research documents, whether they are published or not. The documents may come from teaching and research institutions in France or abroad, or from public or private research centers.
L'archive ouverte pluridisciplinaire HAL, est destinée au dépôt et à la diffusion de documents scientifiques de niveau recherche, publiés ou non, émanant des établissements d'enseignement et de recherche français ou étrangers, des laboratoires publics ou privés. 


\section{Influence of viscoelastic and viscous absorption on ultrasonic wave propagation in cortical bone: application to axial \\ transmission}

Salah Nailia)

Université Paris-Est, Laboratoire de Modélisation et Simulation Multi-Echelle, FRE CNRS 3160, 94010 Créteil Cédex, FRANCE.

Mai-Ba $\mathrm{Vu}^{\mathrm{a})}$

Université Paris-Est, Laboratoire de Modélisation et Simulation Multi-Echelle, FRE CNRS 3160, 94010 Créteil Cédex, FRANCE.

Quentin Grimal ${ }^{\text {b) }}$

Laboratoire d'Imagerie Paramétrique, UMR CNRS 7623, Université Pierre et Marie Curie, 75006 Paris, FRANCE.

Maryline Talmant ${ }^{c)}$

Laboratoire d'Imagerie Paramétrique, UMR CNRS 7623, Université Pierre et Marie Curie, 75006 Paris, FRANCE.

Christophe Desceliers ${ }^{\mathrm{d})}$

Université Paris-Est, Laboratoire de Modélisation et Simulation Multi-Echelle, FRE CNRS 3160, 77454 Marne la Vallée Cédex 2, FRANCE.

Christian Soize ${ }^{\mathrm{e})}$

Université Paris-Est, Laboratoire de Modélisation et Simulation Multi-Echelle, FRE CNRS 3160, 77454 Marne la Vallée Cédex 2, FRANCE.

Guillaume Haïat ${ }^{\mathrm{f})}$

CNRS, Laboratoire de Recherches Orthopédiques, UMR CNRS 7052 B2OA, Université Paris 7, 75010 Paris, FRANCE.

(Dated: 24th June 2009)

Running title: Viscoelastic bone assessed with axial transmission 
a) Electronic address: naili@univ-paris12.fr

b) Electronic address: quentin.grimal@lip.bhdc.jussieu.fr

c) Electronic address: talmant@lip.bhdc.jussieu.fr

d) Electronic address: desceliers@univ-mlv.fr

e) Electronic address: soize@univ-mlv.fr

f) Electronic address: guillaume.haiat@univ-paris-diderot.fr 


\section{Abstract}

Cortical bone and the surrounding soft tissues are attenuating media, which might affect the radiofrequency signals measured with axial transmission devices. Cortical bone is highly heterogeneous, due to a gradient of material properties from the outer to inner part of the cortical shell. The aim of this work is to evaluate the effect of the acoustic absorption in bone and in soft tissues on the ultrasonic response of the bone structure. Therefore, a two-dimensional finite element time domain method is derived to model transient wave propagation in a three-layer medium composed of an inhomogeneous transverse isotropic viscoelastic solid layer sandwiched between two viscous fluid layers. The model couples viscous acoustic propagation in both fluid media with the anisotropic viscoelastic response of the solid. A constant spatial gradient of material properties is considered for two values of bone thicknesses corresponding to a relatively thick and thin bone. Viscous absorption in the surrounding fluid tissues does not affect the results, whereas the viscoelastic properties of bone have a significant effect on the velocity of the First Arriving Signal (FAS). The component of the viscoelastic tensor affecting the results are the same as that of the stiffness tensor found in Haiat et al. (2009). For a thin bone, the FAS velocity is determined by the spatially averaged bone properties. For a thick bone, the FAS velocity may be predicted using a simple signal processing technique; the results allow the derivation of an equivalent penetration depth in the case of a gradient of viscoelasticity, but not in the case of a gradient of porosity.

PACS numbers: 43.80.Ev Acoustical measurement methods in biological systems and media, 43.20.Mv Waveguides, wave propagation in tubes and ducts, 43.20.Px Transient radiation and scattering, 43.40.Rj Radiation from vibrating structures into fluid media, 43.35.Pt Surface waves in solids and liquids, 43.35.Mr Acoustics of viscoelastic materials 


\section{INTRODUCTION}

Different metabolic diseases such as osteoporosis may affect bone quality (WHO Study Group, 1994), resulting in a decrease of bone mass and micro-architectural deterioration of bone tissue which implies an increase of bone fragility. Historically, most ultrasonic studies in the bone field have focused on cancellous bone because the heel, which is mostly composed of cancellous bone, constitutes the most frequently measured anatomical site in the clinic. The diaphysis of long bones such as radius and femur is mainly constituted of cortical bone. Investigating cortical bone quality is of interest (Rico, 1997) because it accounts for about $80 \%$ of the skeleton, supports most of the load of the body and is mainly involved in osteoporotic fractures (Seeley et al., 1991). Moreover, cortical bone quality has recently been shown to be determinant for bone mechanical stability (Mayhew et al., 2005) at the femur.

In axial transmission (AT) technique (which is particularly adapted to cortical bone evaluation), both ultrasound emitter and receivers are placed in the same side of the investigated skeletal site along a direction close to the long bone axis. The earliest event or wavelet (usually called First Arriving Signal, FAS) of the multicomponent signal recorded by the receivers has been the most widely investigated. The wave velocity associated with this signal, which is measured in the time domain, can be used to discriminate healthy subjects from osteoporotic patients and is therefore considered as a relevant index of bone status (Barkmann et al., 2000; Hans et al., 1999; Stegman et al., 1995). Both experimental (Bossy et al., 2004c; Raum et al., 2005) as well as simulation studies (Bossy et al., 2004b) have shown that the FAS velocity was related to different bone properties (bone mineral density, cortical thickness and bone elastic properties). Numerical simulations have been employed to show that when the cortical thickness is comparable or larger than the wavelength, the type of wave contributing to the FAS corresponds to a lateral wave, whereas when the wavelength is larger than the cortical thickness divided by four, the received signal corresponding to the FAS comes from the first symmetric Lamb wave mode $\left(S_{0}\right)$ guided by the cortical thickness (Bossy et al., 2002).

Cortical bone is a complex medium from a biomechanical point of view. Its elastic behavior has been described as transverse isotropic in different works (see for example Dong and Guo (2004); Haiat et al. (2009)). At the macroscopic scale, porosity in the radial direction 
(which is associated with the cross-section of the bone) is heterogeneous at all ages and for both genders (Bousson et al., 2001; Thomas et al., 2005): the mean porosity in the endosteal region (inner part of the bone) is significantly higher than in the periosteal region (outer part of the bone). Moreover, cortical bone is affected by age-related bone resorption and osteoporosis. It undergoes a thinning of the cortical shell, as well as an increase in porosity mainly in the endosteal region (Bousson et al., 2001). An increase of porosity is likely to affect bone material properties (mass density and elasticity) (Fritsch and Hellmich, 2007), which may in turn impact bone quality (Ammann and Rizzoli, 2003). Similarly, a thinning of the cortical shell is an important indicator of decreasing bone strength and of fracture risk (Turner, 2002). Moreover, cortical bone is a strongly attenuating medium where ultrasonic propagation occurs with losses (Han et al., 1996; Lakes et al., 1986; Langton et al., 1990; Lees and Klopholz, 1992; Serpe and Rho, 1996). Ultrasonic attenuation may be due to the viscoelastic behavior of the bone matrix as well as to the presence of the pores (through scattering effects of the ultrasonic wave) (Sasso et al., 2007, 2008). The feasibility of frequency dependent attenuation coefficient measurements has been demonstrated in bovine cortical bone samples of a scale of the centimeter (Sasso et al., 2007). Interestingly, Broadband Ultrasonic Attenuation (BUA, defined as the slope of the curve of the frequency dependent attenuation coefficient) measurements have recently been shown to be significantly related to the microstructure as well as to bone physical properties such as mass density and bone mineral density (Sasso et al., 2008). Therefore, BUA has been evoked as a suitable parameter for cortical bone quality estimation. Similarly, human soft tissues such as skin, fat and muscles (between the transducers and bone) or bone marrow (inside cortical bone) are also media where ultrasonic attenuation has been measured.

The potential advantage of numerical simulation tools over experimental approaches is that it can be used to determine the influence of each bone property independently, which is difficult when working with real samples as all bone geometrical and mechanical properties evolve in parallel. Modeling the FAS in AT experiment is a time-domain elasto-acoustic problem. Time domain analytical methods have been used in the past to solve the elastoacoustic wave system in simple AT models (Grimal and Naili, 2006; Macocco et al., 2005, 2006). Bossy et al. (2004b) have assessed the influence of a gradient of longitudinal wave velocity due to a heterogeneous distribution of porosity on the FAS velocity. More recently, our group has determined (using the Comsol software (COMSOL Multiphysics, 2005)) the effect 
of heterogeneous material bone properties on the ultrasonic response and more specifically on the FAS velocity using 2-D finite element model (FEM) (Haiat et al., 2009). Most models of AT developed in the past have considered cortical bone and the surrounding soft tissues as an elastic material. However, the influence of ultrasonic attenuation (in bone and/or in the surrounding soft tissues) on the ultrasonic response of the investigated anatomical site in the framework of the AT device remains unclear. Studying the influence of attenuation in bone on the FAS velocity is of particular interest since BUA has been suggested as a potential indicator of bone status. In addition, the determination of the sensitivity of the ultrasonic response of bone to attenuation variation may be considered as a first step towards the resolution of the inverse problem.

The aim of this paper is to assess the effect of the viscoelastic nature of cortical bone and of the viscous nature of the surrounding soft tissues on the ultrasonic response obtained with an AT device. Here, bone is modeled as an anisotropic (transverse isotropic) heterogeneous (a gradient of material properties in the radial direction is considered) viscoelastic material and the surrounding soft tissues are modeled as homogeneous viscous liquids. More specifically, we aim at investigating the potentiality of 2-D finite element numerical simulation tools to assess the sensitivity of the FAS velocity to variations of different viscoelastic parameters.

With this introduction as background, a 2-D finite element model briefly described in section II is used to compute the dependence of the FAS velocity on all viscoelastic coefficients of cortical bone at the organ level as well as on the viscous nature of the surrounding soft tissues. The models used for the viscoelastic tensor are presented and discussed. Section III describes i) the sensitivity of the FAS velocity to changes of each viscoelastic coefficient for homogeneous material properties, ii) the effect of a constant gradient of each viscoelastic coefficient affecting the FAS velocity and iii) the effect of a constant gradient of porosity. Results are then discussed in section IV. 


\section{METHOD}

\section{A. Axial transmission configuration}

The geometrical configuration used in the present study is the same as the one used in Haiat et al. (2009). Briefly, cortical bone is modeled as a two-dimensional multilayer medium composed of one heterogeneous viscoelastic transverse isotropic solid layer (corresponding to cortical bone) sandwiched between two homogeneous viscous fluid layers as shown in Fig. 1. The position $\mathbf{x}$ is specified through the Cartesian coordinates $(x, z)$ and time coordinate is denoted by $t$. The upper medium $(z>0)$ corresponds to soft tissues and the lower medium $(z<-h)$ corresponds to bone marrow. Both soft tissues and bone marrow are modelled by viscous fluids. The cortical thickness is denoted $h$ and the direction $z$ corresponds to the bone radial direction. The other sizes of all domains have been chosen in order to avoid any effect of the wave reflected by the boundaries of the domain on all recorded radiofrequency signals. The material properties of soft tissues, bone marrow and cortical bone will be detailed in subsection II C and their spatial variation in subsection II E.

In the simulation, a pressure source is positioned in the fluid and the excitation signal is a Gaussian pulse with a center frequency of $1 \mathrm{MHz}$ identical to the one given in Desceliers et al. (2008). The 14 receivers are regularly spaced with a pitch of $0.8 \mathrm{~mm}$ and a distance of emitter to closest receiver equal to $11 \mathrm{~mm}$. The geometrical arrangement mimics that of an actual probe developed by the 'Laboratoire d'Imagerie Paramétrique' (France) (Bossy et al., 2004a). The FAS velocity is then determined following the procedure used in experiments with the actual probe. Signals are collected for each one of the 14 receivers. The time of the first maximum of the FAS is determined for each signal and inter-sensors delays are then evaluated by computing the time difference between adjacent sensors (Bossy et al., 2004a). The FAS velocity estimate is then given by the slope of the position of each sensor versus time delays, obtained through a least-square linear regression.

\section{B. Two-dimensional governing equations}

In both fluid media, the formulation is written in terms of pressure. Neglecting the body forces and assuming the irrotational motion, the equation of propagation in the viscous fluid 
is given by the wave propagation equation in a viscous media:

$$
\rho_{f} \ddot{p}=\eta_{f} \Delta \dot{p}+K \Delta p
$$

where $p(\mathbf{x} ; t)$ denotes the pressure field, $\rho_{f}$ mass density, $K$ the fluid compressibility and $\eta_{f}$ the bulk viscosity of the fluid. The shear viscosity of the fluid is assumed negligible as compared to that bulk viscosity. Note that the acoustic wave velocity $c_{f}$ in absence of viscosity $\left(\eta_{f}=0\right)$ is given by $c_{f}=\sqrt{\frac{K}{\rho_{f}}}$. The derivatives with respect to the time $t$ are denoted by superposed dot and the Laplace operator is designated by $\Delta$.

In the solid media, the formulation is written in terms of displacement. If no body forces are considered, the momentum conservation equation writes:

$$
\operatorname{div} \boldsymbol{\sigma}=\rho_{s} \ddot{\mathbf{u}},
$$

where $\boldsymbol{\sigma}(\mathbf{x} ; t)$ is the stress tensor, $\rho_{s}$ is the bone mass density, $\mathbf{u}(\mathbf{x} ; t)$ is displacement vector and div designates the usual divergence operator acting on a second-order tensor.

Conversely, the formulation in the solid layer is given in terms of displacement. Cortical bone is modeled as an heterogeneous material using the linear theory of viscoelastic without memory. In this theory, the stress tensor $\boldsymbol{\sigma}$ is linearly related to the strain tensor $\boldsymbol{\epsilon}(\mathbf{x} ; t)$ and to the rate-of-deformation tensor $\dot{\boldsymbol{\epsilon}}(\mathbf{x} ; t)$ :

$$
\boldsymbol{\sigma}=\mathbb{C} \epsilon+\mathbb{E} \dot{\boldsymbol{\epsilon}}
$$

where $\mathbb{C}(\mathbf{x})$ is the stiffness tensor and $\mathbb{E}(\mathbf{x})$ is the viscoelastic tensor. For a 2-D transverse isotropic medium and in the framework of planar deformations, the stiffness tensor writes:

$$
\mathbb{C}=\left(\begin{array}{ccc}
C_{11}(z) & C_{13}(z) & 0 \\
C_{13}(z) & C_{33}(z) & 0 \\
0 & 0 & C_{55}(z)
\end{array}\right)
$$

where all stiffness coefficients, written using the Voigt notation, only depend on $z$ because cortical bone is heterogeneous in the radial direction, similarly as what was done in Haiat et al. (2009).

Cortical bone has been shown to be a significantly anisotropic medium in terms of ultrasonic attenuation as BUA values measured in the axial direction are significantly smaller 
than BUA values obtained in the radial and tangential directions (Sasso et al., 2007). Moreover, no significant difference was found between BUA values measured in the radial and tangential directions (Sasso et al., 2007). Therefore, we have considered an anisotropic (transverse isotropic) tensor $\mathbb{E}$ to describe this dissipative behavior.

Moreover, in a recent study (Sasso et al., 2008), BUA values were found to depend significantly on bone mineral density as well as on mass density, which are two quantities closely related to the porosity. As the spatial distribution of porosity is heterogeneous in cortical bone (Bousson et al., 2001), the viscoelastic properties of cortical bone are also expected to be spatially dependent. Therefore, we have considered an heterogeneous behavior of the viscoelastic tensor $\mathbb{E}$ similar to the one given in Eq. (4) and the viscoelastic tensor $\mathbb{E}$ writes:

$$
\mathbb{E}=\left(\begin{array}{ccc}
\eta_{11}(z) & \eta_{13}(z) & 0 \\
\eta_{13}(z) & \eta_{33}(z) & 0 \\
0 & 0 & \eta_{55}(z)
\end{array}\right)
$$

where all viscoelastic coefficients, written using the Voigt notation, only depend on $z$.

The boundary conditions at the limits of the simulation box are identical to the one used in Desceliers et al. (2008), i.e. stress-free for the solid layer and Neumann conditions for the fluid layers. At both interfaces between the fluid layers and the solid layer, the boundary conditions in terms of displacement and normal stresses are taken into account. The model therefore fully describes the fluid-structure interaction between the three sub-domains, accounting for all reflection, refraction, and mode conversion effects. For each computation, around 186, 000 triangular elements are used, resulting in about 393, 000 degrees of freedom. The simulation software is the 3.5 version of COMSOL Multiphysics (2005). Accurate discretization of the geometrical space is important when simulating wave propagation. The internodal distance in both directions is chosen at least smaller than $0.15 \mathrm{~mm}$ in the fluid and $0.25 \mathrm{~mm}$ in the solid, so that it is lower than the smallest wavelength divided by 6 in all directions and both media. This condition leads to a spatially resolved wave propagation (Moser et al., 1999). The time step used in the computation is of the order of $10^{-3} \mu \mathrm{s}$ and one computation takes about 5 hours on a desk workstation. 


\section{Determination of a realistic range of variation of material properties}

\section{Viscosity of soft tissues and bone marrow}

The mechanical properties of both fluid layers (soft tissues and bone marrow) were considered to be homogeneous in all computations. In addition, we assumed constant values for the mass density $\rho_{f}=1 \mathrm{~g} \cdot \mathrm{cm}^{-3}$ and the compressibility $K=2.25 \times 10^{9} \mathrm{~Pa}$ corresponding to an acoustic wave velocity $c_{f}$ of $1500 \mathrm{~m} \cdot \mathrm{s}^{-1}$ in the absence of absorption $\left(\eta_{f}=0\right)$, close to what has been measured in bone marrow (El Sariti et al., 2006) and in soft tissues.

Acoustic absorption data taken from the literature are used in order to define realistic numerical values for the viscosity $\eta_{f}$ of the soft tissues and of bone marrow. The viscous coefficient $\eta_{f}$ indicated in Eq. (1) is related to the frequency dependent attenuation coefficient of a plane wave $\alpha_{f}$ through the relation (Royer and Dieulesaint, 2000):

$$
\eta_{f}=\frac{2 \alpha_{f} c_{f} K}{\left(\omega^{2}-\alpha_{f}^{2} c_{f}^{2}\right)},
$$

where $\omega$ is the pulsation and $\alpha_{f}$ is the frequency dependent attenuation coefficient. Note that around $f=1 \mathrm{MHz}, \omega=2 \pi f \gg \alpha_{f} c_{f}$, so that Eq. (6) simply writes:

$$
\eta_{f}=\frac{2 \alpha_{f} c_{f} K}{\omega^{2}} .
$$

In our time domain formulation, $\eta_{f}$ is assumed to be independent of frequency and determined by the value of $\alpha_{f}$ at $1 \mathrm{MHz}$, which corresponds to the center frequency of the frequency bandwidth of interest. The constant value of $\eta_{f}$ leads to a squared frequency dependence of the attenuation coefficient.

\section{Stiffness tensor of cortical bone}

In order to define realistic numerical values for the different components of the stiffness tensor of cortical bone and for their variation, the same approach as the one used in Haiat et al. (2009) is used and is briefly recalled in what follows.

We consider in vitro measurements published in Dong and Guo (2004) performed using tensile and torsional tests with a mechanical testing system in 18 different human femoral cortical bone specimens. The homogenized elastic coefficients of the stiffness tensor are constrained to fully verify the thermodynamical conditions of stability. Here, we assume 
that cortical bone is transverse isotropic as this approximation has been shown to be realistic experimentally by different authors (Dong and Guo, 2004; Reilly and Burnstein, 1974; Rho, 1996). Such approximation has been employed in different numerical studies of ultrasonic wave propagation (Bossy et al., 2004b; Haiat et al., 2009; Protopappas et al., 2007).

The values of the stiffness coefficients corresponding to the mean values of the bone mechanical properties are referred to as "reference" set of parameters in what follows. The maximum and minimum values of the stiffness coefficients are obtained by considering respectively the maximum and minimum values of the elastic constants determined in Dong and Guo (2004), after having verified that the thermodynamical stability conditions given by Royer and Dieulesaint (2000) are enforced.

We choose a mean value of mass density $\rho_{s}$ equal to 1.722 g. $\mathrm{cm}^{-3}$, following the value taken in Haiat et al. (2009); Macocco et al. (2006). This value is chosen for the reference mass density. In order to derive a realistic range of variation for mass density, we assume that the reference value is given by a porosity of $7 \%$, which corresponds approximately to the mean porosity at the radius (Baron et al., 2007). The porosity was assumed to vary between 3 and 15\% (Bousson et al., 2001; Dong and Guo, 2004) and a rule of mixture leads to the range of variation of mass density.

\section{Viscoelastic tensor of cortical bone}

The determination of realistic values of the components of the viscoelastic tensor $\mathbb{E}$ is performed by considering the experimental results of ultrasonic attenuation measurements obtained in the literature. To obtain the characteristic value of $\eta_{11}$, we consider a longitudinal plane wave mode propagating in the $x$-axis (axial direction). Then, the displacement $u_{1}$ in the $x$-axis writes:

$$
u_{1}(x, t)=A_{1} \exp i(\omega t-k x),
$$

where $A_{1}$ is the amplitude of the wave at $x=0, k=k_{L, x}-i \alpha_{L, x}$ is the complex wavenumber and $i$ is the imaginary unit. The real part of the complex wavenumber $k$ writes:

$$
k_{L, x}=\frac{\omega}{v_{L, x}^{\phi}}, \quad \text { where } v_{L, x}^{\phi}=\sqrt{\frac{C_{11}}{\rho_{s}}}\left(1+\frac{3 \omega^{2} \eta_{11}^{2}}{8 C_{11}^{2}}\right)
$$

is the longitudinal phase velocity in the $x$-axis (Royer and Dieulesaint, 2000). The quantity $\alpha_{L, x}$ corresponds to the attenuation coefficient of the longitudinal wave mode in the $x$-axis. 
Inserting the relation (8) in (2) and considering an homogeneous transverse isotropic solid, we obtain a simple relationship between the attenuation coefficient $\alpha_{L, x}$ and the viscoelastic coefficient $\eta_{11}$ given by:

$$
\eta_{11}=\frac{2 \alpha_{L, x} C_{11}}{\omega^{2}} \sqrt{\frac{C_{11}}{\rho_{s}}},
$$

under the approximation that $\omega \gg \alpha_{L, x} v_{L, x}^{\phi}$. Equation (10) is similar to Eq. (7).

The same results can be obtained by considering a wave propagation in the $z$ direction (radial direction) given by:

$$
\begin{aligned}
v_{L, z}^{\phi} & =\sqrt{\frac{C_{33}}{\rho_{s}}}\left(1+\frac{3 \omega^{2} \eta_{33}^{2}}{8 C_{33}^{2}}\right) \\
\eta_{33} & =\frac{2 \alpha_{L, z} C_{33}}{\omega^{2}} \sqrt{\frac{C_{33}}{\rho_{s}}}
\end{aligned}
$$

where $\alpha_{L, z}$ denotes the attenuation coefficient in the direction $z$ and $v_{L, z}^{\phi}$ is the longitudinal phase velocity in the $z$-axis.

Using a similar approach, the relation between the attenuation coefficient of the shear wave mode $\alpha_{T}$ and the viscoelastic coefficient $\eta_{55}$ can be obtained by considering a planar transverse wave propagation in the direction $x$ and writes:

$$
\eta_{55}=\frac{2 \alpha_{T} C_{55}}{\omega^{2}} \sqrt{\frac{C_{55}}{\rho_{s}}},
$$

The transverse phase velocity is given by:

$$
v_{T}^{\phi}=\sqrt{\frac{C_{55}}{\rho_{s}}}\left(1+\frac{3 \omega^{2} \eta_{55}^{2}}{8 C_{55}^{2}}\right)
$$

We could not find in the literature a simple way to determine the value of $\eta_{13}$. Therefore, the mean value of $\eta_{13}$ is derived from the mean values of $\eta_{11}$ and $\eta_{55}$ by assuming an isotropic behavior of viscoelasticity, which leads to the relation:

$$
\eta_{13}=\eta_{11}-2 \eta_{55}
$$

In addition, the minimum and maximum values of $\eta_{13}$ were obtained by verifying that the following thermodynamical stability condition:

$$
-\eta_{13}^{2}+\eta_{11} \eta_{33}>0,
$$


is always respected when varying each material property independently. This approach constitutes a simple way of determining a realistic range of variation for $\eta_{13}$. The physiological range of variation of the other viscoelastic constants is obtained by considering the different values of ultrasonic attenuation measured in the literature. In what follows, we will consider a spatial variation of the different viscoelastic properties within this range of variation.

\section{Lamb wave and bulk longitudinal wave}

In previous analysis of wave propagation in idealized bone shapes (plate or tube) made of an elastic transversely isotropic material (Bossy et al., 2002, 2004b; Haiat et al., 2009), the FAS velocity was explicitly related to elastic properties in the limit of large and short wavelength. When the thickness $h$ is smaller than the wavelength $\lambda$ (typically $h / \lambda<0.25$ ) the FAS velocity tends towards the so-called plate velocity which is the phase velocity of the first symmetric Lamb wave mode $S_{0}$ in the large wavelength limit, with the expression:

$$
\mathrm{v}_{\mathrm{p}}=\sqrt{\frac{C_{11}}{\rho_{s}} \times\left(1-\frac{C_{13}^{2}}{C_{11} \times C_{33}}\right)},
$$

which is only valid in the case of an elastic material.

When the thickness $h$ is large compared to wavelength, the FAS velocity tends towards the bulk longitudinal wave velocity inside the material constituting the solid layer:

$$
\mathrm{v}_{\mathrm{b}}=\sqrt{\frac{C_{11}}{\rho_{s}}},
$$

which is again only valid in the case of an elastic material.

In the present study, simulations were performed with two different solid layer thicknesses, $h=0.6 \mathrm{~mm}$ and $h=4 \mathrm{~mm}$ because these two values of $h$ corresponds to cases where the behavior of the FAS velocity have been identified. Considering the dominant frequency of the broadband ultrasonic pulse and the range of variation of $C_{11}$, it means that $h / \lambda$ varies in the intervals defined from 0.14 to 0.19 and from 0.96 to 1.25 respectively for the thin and thick solid layers.

For the thinnest layer, the propagation in the solid layer is analyzed on the basis of the propagation of $S_{0}$ wave in an immersed homogeneous plate after adjustment of the material properties. Therefore, roots associated with the characteristic equation of the $S_{0}$ wave for homogenous transverse isotropic plate are calculated in the limit of large wavelengths. 


\section{E. Modeling a gradient of material property}

Similarly as what was done in Haiat et al. (2009), the impact of a controlled gradient vector $\boldsymbol{\delta}$ of a given material property $S$ on the FAS velocity is investigated. In what follows, the scalar $S$ corresponds to one of the viscoelastic coefficients $\eta_{i j}$ defined in Eq. (5). In each set of simulations, all the material properties (stiffness and viscoelastic coefficients) are constant and equal to their reference value while $S$ is subjected to a gradient defined below.

The gradient vector $\boldsymbol{\delta}=\operatorname{grad} S=\delta \mathbf{z}$ is assumed to be independent of $x$ in all cases, where $\mathbf{z}$ is an unit vector along $z$-axis and grad is the gradient operator acting on a scalar field. The quantity $\delta$ is always taken negative because attenuation increases with porosity (Sasso et al., 2008) and porosity is higher in the endosteal part than in the periosteal part of the bone. Moreover, only the simple situation of affine spatial variations of $S$ is considered, corresponding to a constant value of $\delta$. This affine spatial variation of $S$ is chosen because the actual physiological spatial dependence of $S$ remains unknown. Two different affine spatial dependencies of the studied viscoelastic property are considered and are illustrated in Fig. 2. The associated gradient $\delta$ will be referred to as type 1 or 2 .

Type 1 . The gradient of type 1 is such that the physical property $S$ takes the same value $S_{m}$ at the upper interface $z=0$ of the solid plate for all values of the gradient $\delta$. The quantity $S(z)$ is therefore given by:

$$
S(z)=S_{m}+\delta \times z
$$

where $S_{m}$ is given by the minimal value of the material property $S$ considered. The minimal value $\delta_{m}$ of $\delta$ (which corresponds to its maximum in absolute value as $\delta<0$ ) is chosen so that $S(-h)$ is equal to $S_{M}$, where $S_{M}$ is given by the maximal value of $S$. The gradient $\delta_{m}$ is given by:

$$
\delta_{m}=\frac{\left(S_{m}-S_{M}\right)}{h}<0
$$

Type 2. The gradient of type 2 is such that $S$ takes the same value at the middle of the solid plate $(z=-h / 2)$ for all values of gradient $\delta$. Furthermore, the mean value of the property $S$ is identical for all $\delta$ and the quantity $S(z)$ is given by:

$$
S(z)=\frac{\left(S_{m}+S_{M}\right)}{2}+\delta \times\left(z+\frac{h}{2}\right)
$$

The minimal value of $\delta$ is also given by Eq. (20) so that all values of $S(z)$ are again always comprised between $S_{m}$ and $S_{M}$. 
For both types of spatial variation, five different values of $\delta$ regularly distributed between $\delta_{m}$ and 0 are arbitrarily considered for each layer thickness. In what follows, the notations $\delta_{11}, \delta_{13}, \delta_{33}$ and $\delta_{55}$ correspond to $\delta$ when $S$ is defined by $\eta_{11}, \eta_{13}, \eta_{33}$ and $\eta_{55}$ respectively.

\section{F. Modeling a gradient of porosity}

In the case of bone, all material properties (mass density, stiffness and viscoelastic coefficients) are expected to exhibit coupled spatial variations because they are all related to porosity, which increases from the periosteal to the endosteal part (Bousson et al., 2001). When porosity increases, the values of the homogenized elastic constants and of mass density are expected to decrease, having opposite and competing effects on the wave velocity. In addition, the viscoelastic constants are expected to increase with porosity (Sasso et al., 2008). Here, spatial variations of types 1 and 2 are considered for the porosity (noted $P$ ), with the minimum and maximum values of porosity $P_{m}$ and $P_{M}$ equal respectively to 3 and $15 \%$. In the case of a spatial variation of types 1 and 2 , the porosity writes respectively:

$$
\begin{gathered}
P(z)=P_{M}+\delta_{P} \times z, \\
P(z)=\frac{\left(P_{m}+P_{M}\right)}{2}+\delta_{P} \times\left(z+\frac{h}{2}\right) .
\end{gathered}
$$

The dependence of mass density and of the stiffness coefficients is similar to what has been done in Haiat et al. (2009) and is recalled in what follows. Following a simple rule of mixture, a variation of porosity induces an affine variation of mass density given by:

$$
\rho_{s}(z)=\rho_{m}+\delta_{\rho} \times(P-3)
$$

Here, we choose $\rho_{m}$ in order to obtain a variation of mass density from 1.753 to 1.66 g.cm $\mathrm{cm}^{-3}$ when $P$ varies from 3 and $15 \%$, which leads to $\delta_{\rho}=7.7 \times 10^{-3}$ g.cm ${ }^{-3}$. These values correspond to a mass density equal to 1.722 g.cm ${ }^{-3}$ when $P=7 \%$.

The variations of all material properties with porosity are taken from the literature. Affine dependence of diagonal components of $\mathbb{C}$ with porosity was derived from Baron et al. (2007) where a variation of porosity between 3 and 15\% corresponds approximately to a change of $C_{11}$ and $C_{33}$ of 7.8 and $4 \mathrm{GPa}$, respectively:

$$
C_{11}(z)=C_{11}^{m}+\delta_{C} \times(P-3), \quad C_{33}(z)=C_{33}^{m}+\delta_{C}^{\prime} \times(P-3) .
$$


The variations of $C_{11}$ and $C_{33}$ are centered on their reference value. Therefore, $C_{11}^{m}$ and $C_{33}^{m}$ are respectively equal to 19.7 and $16.85 \mathrm{GPa}$; the quantities $\delta_{C}$ and $\delta_{C}^{\prime}$ are respectively equal to 0.65 and $0.33 \mathrm{GPa}$. Note that taking into account a slight non linear variation of $C_{11}$ and $C_{33}$ as a function of porosity should not modify significantly our results. Similarly as what was done in Haiat et al. (2009), we did not consider any variation of $C_{13}$ which was taken equal to its reference value.

Although BUA has been shown to increase when mass density and bone mineral density (which are both negatively correlated to porosity) increase (Sasso et al., 2008), the precise relationship between porosity and attenuation remains unknown. Therefore, for each viscoelastic constant $\eta_{i j}$, we assume i) a linear relation between the corresponding attenuation at $1 \mathrm{MHz}$ and porosity, ii) that the ultrasonic attenuation value at $P_{m}$ (respectively $P_{M}$ ) corresponds to its minimal (respectively maximal) value within the physiological range. This approach constitutes a simple mean of investigating the effect of viscoelasticity variations due to heterogeneous porosity.

\section{RESULTS}

\section{A. Range of realistic material properties}

\section{Fluid viscosity}

The same approach as the one described in Haiat et al. (2008a) is used to derive the attenuation coefficient in bone marrow because experimental measurements could not be found in the literature. Therefore, we assumed that the absorption properties of bone marrow are close to that of fat (soft tissue like behaviour) and the values of $\eta_{f}$ used for the soft tissues and for bone marrow are therefore similar. The attenuation coefficient at $1 \mathrm{MHz}$ measured by Dussik and Fritch (1956) in fresh human fat tissues is equal to $0.8 \mathrm{~dB} . \mathrm{cm}^{-1}$ and that measured by Lehman and Johnson (1958) in subcutaneous pig fat tissue is equal to $1.8 \mathrm{~dB} \cdot \mathrm{cm}^{-1}$ (these values are summed up in Goss et al. (1978)). As indicated in Table I, the mean value of $\alpha_{L}$ was taken equal to $1 \mathrm{~dB} \cdot \mathrm{cm}^{-1}$ and its minimum and maximum values were arbitrarily chosen equal to 0 and $2 \mathrm{~dB} \cdot \mathrm{cm}^{-1}$ respectively. The corresponding values of $\eta_{L}$ were obtained using Eq. (7). 


\section{Stiffness tensor of cortical bone}

The values used for the stiffness tensor of cortical bone are the same as the one given in Haiat et al. (2009). Table II shows the maximum, minimum and mean values of the 4 components $\left(C_{11}, C_{13}, C_{33}\right.$ and $\left.C_{55}\right)$ of the stiffness tensor $\mathbb{C}$ and of mass density of cortical bone. As described in Haiat et al. (2009), these values were obtained by considering the results obtained in Dong and Guo (2004) and by verifying that the thermodynamical stability equations are respected.

\section{Viscoelastic tensor of cortical bone}

The values of the different components of the viscoelastic tensor $\mathbb{E}$ were obtained by considering the measurement of different attenuation coefficients given in the literature. The values of the longitudinal attenuation coefficients corresponding to the axial and radial directions $\left(\alpha_{L, x}\right.$ and $\left.\alpha_{L, z}\right)$ at $1 \mathrm{MHz}$ were obtained from the axial and radial BUA values respectively, assuming a linear frequency dependence of the attenuation coefficient within the entire frequency bandwidth. The values of $\alpha_{L, x}$ and $\alpha_{L, z}$ given in Table III were taken from Table 1 of Sasso et al. (2007). The mean value of $\alpha_{T}$ indicated in Table III was taken from Garcia et al. (1978). The corresponding values of $\eta_{55}$ was obtained using Eq. (13) by considering the reference values of the material properties indicated in Table II. The mean value of $\eta_{13}$ was obtained using Eq. (15) and the maximum and minimum values of $\eta_{13}$ were obtained by verifying that the thermodynamical stability condition given by Eq. (16) is always respected when $\eta_{11}$ and $\eta_{33}$ vary within their physiological range.

\section{B. Analytical validation of the finite element model}

In order to validate our simulation code in the framework of a viscoelastic constitutive law, the results obtained numerically in a simple geometrical situation (planar propagation) are compared to analytical results. Therefore, a through transmission experiment was simulated using the numerical simulation tool in order to verify that the material properties used as input parameters could be retrieved in the simulation. Briefly, a 2-D plane wave propagation was considered in a rectangular simulation domain $(10 \times 5 \mathrm{~mm})$ in the $x$-axis. A linear emitter was positioned at $x=0$, generating a broadband ultrasonic signal similar to that described in 
subsection II A. A linear receiver is located at the other end of the simulation domain (at $x=$ $10 \mathrm{~mm}$ ) to record the ultrasonic wave after its propagation in the viscoelastic domain where the mean stiffness coefficient indicated in Table II and the viscoelastic parameters indicated in Table III were considered. Longitudinal and transverse wave modes were successively tested by considering a time-dependent displacement in the $x$ and $z$ directions at the emitter as boundary conditions.

Meanwhile, the propagation of a longitudinal ultrasonic wave in water was simulated using the formulation in terms of pressure, leading to the reference signal, which is necessary in the framework of a through transmission configuration. The black solid lines in Fig. 3 show the behavior of the frequency dependent attenuation coefficient and of phase velocity within the frequency bandwidth of interest, obtained using the ratio of the spectra of the reference signal and of the signal transmitted in the simulation domain for the longitudinal and transverse wave modes (Haiat et al., 2008b; Sasso et al., 2008).

These last simulation results were compared to analytical results obtained by considering a plane wave propagation in a viscoelastic medium under the same assumptions of weak absorption given in subsection II C 3 where i) the attenuation coefficient varies has a f-square dependence, as indicated by Eqs. (10) and (13) and ii) the longitudinal and transverse phase velocity $v_{L, x}^{\phi}$ and $v_{T}^{\phi}$ varies as a second order polynomial as a function of frequency, as indicated by Eqs. (9) and (14). The gray dashed lines in Fig. 3 shows the frequency dependencies of the attenuation coefficient and of phase velocity obtained using the analytical model described above. A good agreement between analytical and numerical results is obtained for the frequency dependence of $\alpha_{L, x}$ and $\alpha_{T}$ as the maximum difference between the two results is equal to $0.01 \mathrm{~dB} . \mathrm{cm}^{-1}$ for $\alpha_{L, x}$ and $0.012 \mathrm{~dB} . \mathrm{cm}^{-1}$ for $\alpha_{T}$. Slightly more important discrepancies are obtained between the analytical and the numerical model for the frequency dependence of phase velocity as the difference between the two results slightly increases with frequency and its maximum value is equal to $0.7 \mathrm{~m} . \mathrm{s}^{-1}$ for $v_{L, x}^{\phi}$ and to $0.3 \mathrm{~m} . \mathrm{s}^{-1}$ for $v_{T}^{\phi}$. This discrepancy may be explained by effects related to numerical dispersion in the simulation code. 


\section{Effect of viscous and viscoelastic absorption on the amplitude of the first arriving wavefront}

In order to assess the qualitative effect of viscous properties of both fluids and of the viscoelastic properties of the solid on the FAS, Fig. 4 shows the amplitude of the first maximum of each signal recorded by the 14 receivers corresponding to the computation of the FAS velocity for a bone thickness equal to $4 \mathrm{~mm}$. The values of the components of the stiffness tensor were taken equal to their reference value indicated in Table II. The solid line corresponds to the normalized amplitude of the signal obtained without any absorption neither in the fluid nor in the solid. The dashed line corresponds to the normalized amplitude of the signal obtained by considering absorption in both fluids and in the solid, the viscous and viscoelastic parameters being equal to their reference values given in Table I and III.

The difference between the dashed and solid lines illustrates the influence of viscous and viscoelastic effects on the amplitude of the FAS. For both configurations (elastic and viscous case), the amplitude of the signal is shown to decrease as a function of the position of the receiver, which can be explained by the fact that the wave radiates in water while it propagates in the bone structure, leading to a loss of energy. As shown in Fig. 4, including viscous and viscoelastic absorption in the simulation model leads to a decrease of the amplitude of the first arriving wavefront.

The FAS velocity obtained without absorption is equal to $3630 \mathrm{~m} \cdot \mathrm{s}^{-1}$ whereas it is equal to $3732 \mathrm{~m} . \mathrm{s}^{-1}$ when absorption is taken into account. Note that The FAS velocity is determined by the spatially averaged bone properties. The difference between these two values is significant and will be investigated in what follows.

\section{Dependence of the FAS velocity to changes of viscous properties of the surrounding fluids}

From the analysis of numerical simulations, the variation of the FAS velocity due to changes of viscous properties of the soft tissues and of bone marrow within a realistic range was assessed for $h=0.6$ and $4 \mathrm{~mm}$ and for homogeneous material properties. The material properties of cortical bone were taken equal to their reference values as given in Table II for the stiffness tensor $\mathbb{C}$ and in Table III for the viscoelastic tensor $\mathbb{E}$. The minimal (m) 
and maximal (M) values of $\eta_{f}$ are tested for the soft tissues and for bone marrow, the other material properties remaining equal to their reference values.

Table IV shows that the FAS velocity is independent of the viscous properties of the soft tissues as well as of marrow within the physiological range. Therefore, the soft tissues as well as bone marrow will be considered as non viscous fluids in what follows.

\section{E. Dependence of the FAS velocity to changes of homogeneous bone viscoelastic properties}

The variation of the FAS velocity due to changes of the viscoelastic parameters of cortical bone within a realistic range was assessed for $h=0.6$ and $4 \mathrm{~mm}$ and for homogeneous material properties in order to determine which viscoelastic parameters play a role in the FAS velocity and must thus be considered for spatial variations. Therefore, the material properties of the surrounding soft tissues and of the stiffness tensor were taken equal to their reference values as indicated in Tables I and II. The minimal (m) and maximal (M) values of each component of the viscoelastic tensor $\mathbb{E}$ of cortical bone indicated in Table III were tested, the other material properties remaining equal to their reference values. Table $\mathrm{V}$ shows the FAS velocity variations corresponding to a variation of each material property.

According to the value of $h$ and to the considered viscoelastic property $S$, two situations may be distinguished: the difference of the FAS velocities obtained when considering the maximum and minimum value of $S$ may be relatively "large" (above $70 \mathrm{~m} . \mathrm{s}^{-1}$ ) or relatively "small" (lower than $\left.3 \mathrm{~m} \cdot \mathrm{s}^{-1}\right)$. Considering the thick solid layer $(h=4 \mathrm{~mm}$ ), Table V shows that small differences of the FAS velocity is obtained, when $\eta_{13}, \eta_{33}$ and $\eta_{55}$ take their minimum or maximum values defined above. On the contrary, the FAS velocity varies significantly when $\eta_{11}$ varies within the limits defined above.

The solid line of Fig. 5 shows the variation of the FAS velocity as a function of $\eta_{11}$ in the case of homogeneous bone properties, the other components of $\mathbb{E}$ and $\mathbb{C}$ remaining constant and equal to their reference value. The FAS velocity is shown to be an increasing function of $\eta_{11}$ within the physiological range.

When considering the thin solid layer $(h=0.6 \mathrm{~mm})$, changes in $\eta_{55}$ weakly affects the FAS velocity while variations of $\eta_{11}, \eta_{13}$ and $\eta_{33}$ lead to significantly larger changes in the FAS velocity. As shown in Fig. 6, the FAS velocity is an increasing function of $\eta_{11}$ and 
$\eta_{33}$ and a decreasing function of $\eta_{13}$ within the physiological range. The component of the viscoelastic tensor $\left(\eta_{11}, \eta_{13}\right.$ and $\left.\eta_{33}\right)$ which influence the FAS velocity are the same than the components of the stiffness tensor $\left(C_{11}, C_{13}\right.$ and $\left.C_{33}\right)$ having an effect on the FAS velocity (Haiat et al., 2009) and determining the plate velocity $\mathrm{v}_{\mathrm{p}}$ (see Eq. (17)).

Figure 7 shows the variation the FAS velocity for homogeneous cortical bone where the porosity is assumed to vary within the physiological range, inducing a simultaneous variation of mass density and of the components of $\mathbb{C}$ and of $\mathbb{E}$. The black line of Fig. 7 shows that the FAS velocity is a decreasing function of the porosity for a thick and a thin bone width and that the dependence of the FAS velocity on the porosity is approximately similar.

In what follows, the effect of a gradient of viscoealstic property playing a role in the determination of the FAS velocity will be investigated for each value of the bone thickness.

\section{F. Effect of a gradient of bone viscoelastic properties}

\section{Case of thick solid layer}

Figure 8 shows the dependence of the FAS velocity on the gradient of $\eta_{11}$ in the case of a thick solid layer $(h=4 \mathrm{~mm})$.

When the gradient is of type 1 , the FAS velocity increases when $\delta_{11}$ increases, whereas when gradient is of type 2 , the FAS velocity slightly decreases with $\delta_{11}$. The dashed lines of Fig. 8 show the FAS velocity obtained when considering a constant homogeneous value of $\eta_{11}$ equal to its spatially averaged values, which corresponds to the value of $\eta_{11}$ at $z=-0.5 h$. The dashed lines of Fig. 8 were derived from the results shown in Fig. 5. A poor agreement is obtained between the dashed and solid lines, which shows that in the case of a thick bone width with heterogeneous bone properties, the FAS velocity can not be obtained by simply spatially averaging $\eta_{11}$. These results will be discussed in subsection IV B.

\section{Case of thin solid layer}

When considering the thin solid layer $(h=0.6 \mathrm{~mm})$, the effect of a gradient of $\eta_{11}, \eta_{13}$ and $\eta_{33}$ was investigated according to the results presented in subsection III E and the results are shown in Fig. 9.

When the gradient is of type 1 , the FAS velocity is shown to increase when $\delta_{11}$ and $\delta_{33}$ 
increases whereas it decreases when $\delta_{13}$ increases. When the gradient is of type 2 , the FAS velocity stays approximately constant with $\delta_{11}, \delta_{13}$ and $\delta_{33}$.

The dashed lines of Fig. 9 show the FAS velocity obtained when considering a constant homogeneous value of $\eta_{11}, \eta_{33}$ and $\eta_{13}$ equal to its spatially averaged values, which corresponds to the value of $\eta_{11}, \eta_{33}$ and $\eta_{13}$ at $z=-0.5 h$. The dashed lines of Fig. 8 were derived by considering the results shown in Fig. 5. For both types of gradient, the FAS velocity is shown to be accurately predicted by considering the spatially averaged values of $\eta_{11}, \eta_{33}$ and $\eta_{13}$, which show that in the case of a thin bone width with heterogeneous bone properties, the FAS velocity is governed by the spatially averaged values of the viscoelastic properties $\eta_{11}, \eta_{13}$ and $\eta_{33}$.

\section{G. Effect of a gradient of porosity}

Figure 10(a) and (b) shows the variation of the FAS velocity obtained for $h=0.6$ and $4 \mathrm{~mm}$ respectively when considering a gradient of porosity $\delta_{P}$. The black and gray solid lines show the FAS velocity obtained numerically for a gradient of porosity of types 1 and 2 , respectively. Note that the porosity induces a variation of mass density as well as of the stiffness and viscoelastic coefficients, as described in section II F. The dashed lines of Fig. 10 reproduces the results obtained in Haiat et al. (2009) corresponding to the results obtained by only accounting for variations of stiffness coefficients and of mass density due to changes of porosity.

As shown in Fig. 10, taking into account the viscoelastic behavior of cortical bone induces a significant increase of the FAS velocity compared to the elastic case. In the case of a thin bone width (see Fig. 10(a)), the FAS velocity does not depend on $\delta_{P}$ for a gradient of type 2 , which is a result similar to what has been obtained in the elastic case. However, for a gradient of type 1 , the FAS velocity decreases with $\delta_{P}$ with a slope approximately divided by two compared to the elastic case. The thin dotted line in Fig. 10(a) shows the FAS velocity obtained when considering a constant homogeneous value of the porosity equal to its spatially averaged values, which corresponds to the value of the porosity at $z=-0.5 h$. A good agreement is obtained between the results obtained with a gradient and with spatially averaged material properties, which confirms that in the case of a thin bone width with heterogeneous porosity, the FAS velocity is governed by the spatially averaged value of the 
porosity.

In the case of a thick bone width (see Fig. 10(b)), the FAS velocity increases with $\delta_{P}$ for a gradient of type 2, with a slope slightly lower than what is obtained in the elastic case. However, for a gradient of type 1, the FAS velocity slightly increases with $\delta_{P}$ whereas it decreases with $\delta_{P}$ in the elastic case. These results will be discussed in subsection IV B.

\section{DISCUSSION}

To the best of our knowledge, this study is the first one to focus on the effect of ultrasonic attenuation on the FAS velocity estimated with an axial transmission configuration for different solid layer thicknesses.

Recently, a study where attenuation was neglected has shown that the overlying soft tissue significantly influence the ultrasonic response of cortical bone in axial transmission (Moilanen et al., 2008). The present study shows that ultrasonic attenuation effects are not likely to modify their conclusions. However, as shown in Fig. 4, attenuation effects strongly influence the amplitude of the signal corresponding to the FAS velocity, which is determined using an appropriate threshold. This threshold is usually chosen empirically, by finding a compromise between the noise level and the amplitude of the FAS. Our results show that in the case of bone with strong attenuation, the amplitude of the FAS may be significantly reduced compared to the elastic case (a factor 3 may be expected). This decrease might lead to wrong estimation of the time of the FAS velocity if the FAS amplitude crosses the threshold, which is more likely for the receivers located far from the emitter because losses of amplitude are comparatively more important.

The results shown in Table $\mathrm{V}$ show that the effect of changes of viscoelastic properties within their estimated physiological range may be of the same order of magnitude as the effect of changes of the stiffness coefficient or mass density within the physiological range (Haiat et al., 2009). These results indicate that attenuation is an important property which should be accounted for when modeling the ultrasonic propagation in cortical bone in the context of axial transmission. 


\section{A. Prediction of the influence of viscoelasticity using a signal processing technique}

In order to understand the increase of the FAS velocity as a function of the longitudinal attenuation for both values of bone thickness $h$, an approach using signal processing techniques similar to what has been developed in Haiat et al. (2006) has been applied by assuming the propagation of a single contribution. This approach is based on results obtained in the context of trabecular bone studies showing that speed of sound values measured using a marker in the time domain increases when BUA increases (Haiat et al., 2005). This phenomenon is due to a spreading of the normalized signal when BUA increases (Haiat et al., 2006; Wear, 2000, 2001).

Briefly, we employed a filter with a quadratic frequency dependent attenuation coefficient. Signal simulation was performed in order to understand the effects of the frequency dependent attenuation coefficient on the artifacts in velocity measurements. For a given quadratic frequency-dependent attenuation coefficient in bone $\alpha(f)=\beta f^{2}$, a transfer function was determined so that the effect of the propagation in bone could be modeled as a quadratic filter. In this last relation, $\beta$ is a constant.

In the model, the simulated signal $s_{s}(t)$ (with Fourier transform $S_{s}(f)$ ) corresponding to a propagation in bone over a distance of $L$, was derived from the emitted signal $e(t)$ (Fourier transform $E(f))$ through:

$$
S_{s}(f)=E(f) \exp \left(-\beta f^{2} L\right) \exp \left(-2 i \pi f \frac{L}{v_{L, x}^{\phi}(f)}\right),
$$

where the transfer function $H_{1}(f)=\exp \left(-\beta f^{2} L\right)$ accounts for the attenuation in bone (attenuation in water was taken to be negligible as it does not impact the FAS velocity) and $H_{2}(f)=\exp \left(-2 i \pi f \frac{L}{v_{L, x}^{\phi}(f)}\right)$ accounts for the time delay corresponding to the propagation in bone. Following Eqs. (9) and (10) together with the expression of the attenuation coefficient, we have:

$$
v_{L, x}^{\phi}(f)=\sqrt{\frac{C_{11}}{\rho_{s}}}\left(1+\frac{3 C_{11} \beta}{8 \pi^{2} \rho_{s}}\right) .
$$

For each value of $\beta$, the approach described above allows to construct a set of 14 signals by varying the value of $L$ accordingly to the position of the receivers. A value of $L=0$ was arbitrarily chosen for the receiver located at the left of Fig. 1. Note that choosing another value would not impact the results presented below as the FAS velocity is derived from the 
comparison between the 14 receivers and does not depend on the shape of the first signal. The FAS velocity was derived following the same method as described in subsection II A.

The relationship between the parameter $\beta$ and the bone viscoelastic properties depends on the bone thickness considered. In the case of a large bone thickness, the situation is relatively simple because only $\eta_{11}$ impacts the FAS velocity and using Eq. 10 leads to:

$$
\beta=2 \frac{\eta_{11} \omega^{2}}{C_{11}} \sqrt{\frac{\rho_{s}}{C_{11}}}
$$

The dotted line of Fig. 5 shows the variation of the FAS velocity as a function of $\eta_{11}$. A reasonable agreement is shown between the results obtained using finite element analysis and this signal processing approach, the discrepancy being related to the simplicity of the model. Note that similar results are obtained in the case of a thin bone width (data not shown) when considering an isotropic behavior of $\mathbb{C}$ and $\mathbb{E}$, which is necessary in order to apply this simple model a simple relationship between $\beta$ and the bone material properties is difficult to obtain in the anisotropic case.

\section{B. Contributing depth for thick solid layer}

In a previous study (Haiat et al., 2009), we have shown that an equivalent penetration depth corresponding to the average thickness investigated by the lateral wave could be determined in the case of a large bone thickness. For spatial variations of $C_{11}$ or $\rho_{s}$, the equivalent penetration depth was shown to be independent from the type of gradient, but to depend on the material property considered.

\section{Spatial variation of $\eta_{11}$}

In the case of a thick layer $(h>\lambda)$, the variation of the FAS velocity as a function of a spatial gradient of $\eta_{11}$ obtained in Fig. 8 was analyzed. For this purpose, the notion of contributing depth $H_{11}$ is introduced by adapting the method described in Haiat et al. (2009).

The method described hereafter aims at estimating the value of this equivalent depth $H_{x}$ in the case of a linear spatial variation of $\eta_{11}$, which is the only viscoelastic parameter affecting the FAS velocity when $h=4 \mathrm{~mm}$ (see Table V). The determination of $H_{x}$ is 
important in order to determine which part of the bone is assessed when employing axial transmission techniques.

The methods employed to define the contributing depth $H_{x}$ uses the relationship between the FAS velocity and $\eta_{11}$ in the case of homogeneous material properties, noted $\mathrm{v}_{F}^{x}\left(\eta_{11}\right)$ in what follows. The function $\mathrm{v}_{F}^{11}$ is given in Fig. 5 .

In the case of a gradient of types 1 and $2, \eta_{11}$ depends on $z$ (see Eqs. (19) and (21)), so that we can define a function $\mathrm{v}_{\mathrm{a}}$ of the variables $\delta_{11}$ and $z$ given by:

$$
\mathrm{v}_{\mathrm{a}}\left(\delta_{11}, z\right)=\mathrm{v}_{\mathrm{F}}^{x}\left(\eta_{11}(z)\right)
$$

Note that $\mathrm{v}_{\mathrm{a}}$ can be completely determined within the physiological range for each type of gradient using Fig. (5) and Eqs. (19) and (21).

The contributing depth $H_{x}$ is defined as the depth at which the rate of variation of $\mathrm{v}_{\mathrm{a}}$ computed at the depth $H_{x}$ and of the FAS velocity (obtained numerically) versus $\delta_{11}$ are equal. This condition writes:

$$
\alpha \equiv \frac{d \mathrm{v}_{\mathrm{F}}}{d \delta_{11}}\left(\delta_{11}\right)=\frac{\partial \mathrm{v}_{\mathrm{a}}}{\partial \delta_{11}}\left(\delta_{11}, H_{11}\right)
$$

where $\mathrm{v}_{\mathrm{F}}$ denotes the FAS velocity plotted in Fig. 8.

This method is similar to the one developed in Haiat et al. (2009), except that we previously used an analytical model to determine $\mathrm{v}_{\mathrm{a}}$, which is not possible in the present work.

In the case of a spatial variation of types 1 and 2 of $\eta_{11}$, Eq. (30) yields respectively:

$$
\begin{aligned}
& H_{x}^{1}=\alpha\left(\frac{d \mathrm{v}_{\mathrm{F}}^{x}}{d \eta_{11}}\right)^{-1} \\
& H_{x}^{2}=\frac{h}{2}+\alpha\left(\frac{d \mathrm{v}_{\mathrm{F}}^{x}}{d \eta_{11}}\right)^{-1},
\end{aligned}
$$

where $H_{x}^{1}$ and $H_{x}^{2}$ are the equivalent penetration depths for a spatial variation of $\eta_{11}$ corresponding to a variation of types 1 and 2 respectively. The value of $\alpha$ is determined graphically as the slope of the FAS velocity versus $\delta_{11}$ in Fig. 8. The value of $d \mathrm{v}_{\mathrm{F}}^{x} / d \eta_{11}$ is determined from the plots shown in Fig. 5.

Table VI compares the results obtained in the present study with those obtained in Haiat et al. (2009). The values obtained for $H_{11}$ for a gradient of types 1 and 2 are of the same order of magnitude, which constitutes a further validation of our approach. The results show that the contributing depth corresponding to a gradient of $\eta_{11}$ is higher than that corresponding to a gradient of $C_{11}$ and $\rho_{s}$. 


\section{Spatial variation of porosity}

Similarly as what was done in Haiat et al. (2009), we tried to compute an equivalent penetration depth $H_{P}$ in the case of a gradient of porosity, which induces a simultaneous spatial variation of $\eta_{11}, C_{11}$ and $\rho_{s}$. The computation of $H_{P}$ may be performed similarly as in the last paragraph and requires using the relationship between the FAS velocity and $P$ for homogeneous materials shown in Fig. 7 which is denoted $\mathrm{v}_{\mathrm{F}}^{P}(P)$ in what follows.

In the case of a gradient of types 1 and 2, $P$ depends on $z$ (see Eq. (23)), so that it is possible to formally define a function $\mathrm{v}_{\mathrm{a}}$ of $\delta_{P}$ and $z$ given by:

$$
\mathrm{v}_{\mathrm{a}}\left(\delta_{P}, z\right)=\mathrm{v}_{\mathrm{F}}^{P}(P(z)) .
$$

Note that $\mathrm{v}_{\mathrm{a}}$ is known within the physiological range from the curves plotted in Fig. 7 .

The contributing depth $H_{P}$ is defined as the depth at which the rate of variation of $\mathrm{v}_{\mathrm{a}}$ computed at the depth $H_{P}$ and of the FAS velocity (obtained numerically) versus $P$ are equal. This condition writes:

$$
\alpha \equiv \frac{d \mathrm{v}_{\mathrm{F}}}{d P}\left(\delta_{P}\right)=\frac{\partial \mathrm{v}_{\mathrm{a}}}{\partial \delta_{P}}\left(\delta_{P}, H\right)
$$

where $\mathrm{v}_{\mathrm{F}}$ denotes the FAS velocity plotted Fig. 10(b).

In the case of a spatial variation of types 1 and 2 of $P$, Eq. ((34)) yields respectively:

$$
\begin{aligned}
& H_{P}^{1}=\alpha\left(\frac{d \mathrm{v}_{\mathrm{F}}^{P}}{d P}\right)^{-1}, \\
& H_{P}^{2}=\frac{h}{2}+\alpha\left(\frac{d \mathrm{v}_{\mathrm{F}}^{P}}{d P}\right)^{-1},
\end{aligned}
$$

where $H_{P}^{1}$ and $H_{P}^{2}$ are the equivalent depths for a spatial variation of porosity corresponding to a variation of types 1 and 2 respectively. The value of $\alpha$ is determined graphically as the slope of the FAS velocity versus $\delta_{P}$ in Fig. 10. The value of $d \mathrm{v}_{\mathrm{F}}^{P} / d P$ is determined from the results shown in Fig. 7.

Table VI shows the results derived from Fig. 10 for $H_{P}^{1}$ and $H_{P}^{2}$, which are significantly different. In addition, the value of $H_{P}^{1}$ is negative, which is not a physically acceptable results. These results show that the approach described above is not adapted to define an equivalent penetration depth in the case of a gradient of porosity when taking into bone viscoelastic behavior. Note that in the elastic case, the values of $H_{P}^{1}$ and $H_{P}^{2}$ were also 
different, but to a lesser extent. These results can be explained by the fact that the method described above is based on a first order approximation, which is not valid when all three parameters $\left(\eta_{11}, C_{11}\right.$ and $\left.\rho_{s}\right)$ vary simultaneously. In particular, the negative value obtained for $H_{P}^{1}$ can be explained by the fact that the FAS velocity decreases with porosity in the homogeneous case, whereas it slightly increases with a gradient of type 1 of porosity. In the case of a gradient of type 1 , the contribution of spatial variations of $\eta_{11}$ (corresponding to an increase of the FAS velocity) dominates that of the elastic properties $C_{11}$ and $\rho_{s}$ (corresponding to an decrease of the FAS velocity). This result can be explained by the fact that $H_{P}^{1}$ in the elastic case is lower than $H_{x}^{1}$, leading to a stronger dependence of the FAS velocity on spatial variations of $\eta_{11}$ than on $C_{11}$ and $\rho_{s}$. In addition, the increase of the FAS velocity versus porosity obtained in the case of a gradient of type 1 can be predicted when averaging the FAS velocity obtained when only $\eta_{11}$ varies and when $C_{11}$ and $\rho_{s}$ vary (data not shown).

\section{Limitations}

This study has limitations. First, the determination of homogenized viscoelastic material properties of cortical bone is a complex problem due to the multiscale nature of bone. Bone material properties depend on the microstructure (e.g. porosity, shape and distribution of the pores) as well as on the material properties of the bone matrix at smaller scales (e.g. mineralization, orientation of collagen fibrils, etc.). Both porosity and material properties of bone tissue may depend on the radial position in bone and result in a gradient of all components of $\mathbb{C}, \mathbb{E}$ and in $\rho_{s}$. Therefore multiscale models, coupled with structural and stiffness measurements at lower scales (such as nanoindentation (Zysset et al., 1999) or scanning acoustic microscopy (Raum et al., 2006)) are needed in order to derive more realistic spatial variations of homogenized material properties.

Second, although the range of variation chosen for each component of the viscoelastic tensor was determined from the experimental results obtained by the group of some of the authors Sasso et al. $(2007,2008)$ for bovine cortical bone samples at $4 \mathrm{MHz}$, the precise relationship between the attenuation coefficient and porosity in human cortical bone around $1 \mathrm{MHz}$ remains poorly understood. The values of $\eta_{11}$ and $\eta_{33}$ were obtained by considering BUA values measured at $4 \mathrm{MHz}$ and a linear dependence of the attenuation coefficient 
between 0 and $4 \mathrm{MHz}$. However, the dependence of the attenuation coefficient has been shown to be slightly non linear over a wide frequency bandwidth (see Fig. 5 of Sasso et al. (2007)). Moreover, as shown by Eq. (10), our time model approximation leads to a f-square dependence of the attenuation coefficient, which is not necessarily the case for real cortical bone samples. In order to account for other frequency dependence of the attenuation coefficient in the framework of a time-domain model, would need to be used, similarly as what was done in fluids using a fractional time derivative (Wismer, 2006) or a causal convolution wave equation (Cobbold et al., 2004). However, the frequency dependence of the attenuation coefficient in cortical remains poorly understood. Ultrasonic attenuation has been shown to be related to scattering effects due to the presence of the pores (Sasso et al., 2008). However, viscoelastic properties of the bone matrix are also expected to influence ultrasonic attenuation, but this dependence remains to be quantified. The coupling of scattering and viscous absorption effects makes the prediction of the frequency dependence of the attenuation coefficient difficult. The development of homogenization models capable of predicting bone attenuation and/or dispersive effects, such as the ones developed in the context of trabecular bone would be of great interest to predict the evolution of bone ultrasonic response with age or thickness.

\section{Acknowledgement}

This study was supported by the 'Agence Nationale de la Recherche' (Contract BoneChar number BLAN06-2_144779). 


\section{References}

Ammann, P. and Rizzoli, R. (2003), "Bone strength and its determinants," Osteoporos. Int. 14(Suppl. 3), S13-S18.

Barkmann, R., Kantorovich, E., Singal, C., Hans, D., Genant, H. K., Heller, M., and Gluer, C. C. (2000), "A new method for quantitative ultrasound measurements at multiple skeletal sites," J. Clin. Densitometry 3, 1-7.

Baron, C., Talmant, M., and Laugier, P. (2007), "Effect of porosity on effective diagonal stiffness coefficients $\left(c_{i i}\right)$ and anisotropy of cortical at $1 \mathrm{MHz}$ : A finite-difference time domain study," J. Acoust. Soc. Am. 122, 1810-1817.

Bossy, E., Talmant, M., and Laugier, P. (2002), "Effect of bone cortical thickness on velocity measurements using ultrasonic axial transmission: a 2D simulation study," J. Acoust. Soc. Am. 112(1), 297-307.

Bossy, E., Talmant, M., and Laugier, P. (2004a), "Bi-directional axial transmission can improve accuracy and precision of ultrasonic velocity measurement in cortical bone: a validation on test material," IEEE Trans. Ultrason. Ferroelectr. Freq. Control. 51(1), 7179.

Bossy, E., Talmant, M., and Laugier, P. (2004b), "Three-dimensional simulations of ultrasonic axial transmission velocity measurement on cortical bone models," J. Acoust. Soc. Am. 115(5), 2314-2324.

Bossy, E., Talmant, M., Peyrin, F., Akrout, L., Cloetens, P., and Laugier, P. (2004c), "An in vitro study of the ultrasonic axial transmission technique at the radius: $1-\mathrm{MHz}$ velocity measurements are sensitive to both mineralization and intracortical porosity," Journal of Bone and Mineral Research 19(9), 1548-1556.

Bousson, V., Meunier, A., Bergot, C., Vicaut, E., Rocha, M. A., Morais, M. H., LavalJeantet, A. M., and Laredo, J. D. (2001), "Distribution of intracortical porosity in human midfemoral cortex by age and gender," J. Bone Miner. Res. 16(7), 1308-1317.

Cobbold, R., Sushilov, N., and Weathermon, A. (2004), "Transient propagation in media with classical or power-law loss." J. Acoust. Soc Am. 116(6), 3294-303.

COMSOL Multiphysics (2005), "Model library," Grenoble (France).

Desceliers, C., Soize, C., Grimal, Q., Haïat, G., and Naili, S. (2008), "Three dimensional transient elastic waves in multilayer semi-infinite media solved by a time-space-spectral 
numerical method," Wave Motion 45(4), 383-399.

Dong, X. N. and Guo, X. E. (2004), "The dependence of transversely isotropic elasticity of human femoral cortical bone on porosity," J. Biomech. 37(8), 1281-1287.

Dussik, K. and Fritch, D. (1956), "Deterimination of sound attenuation and sound velocity in the structure constituting the joints, and of the ultrasonic field distribution with the joints of living tissues and anatomical preparations, both in normal and pathological conditions," Tech. rep.

El Sariti, A., Evans, J., and Truscott, J. (2006), "The temperature dependence of the speed of sound in bovine bone marrow at 750 kHz," Ultrasound. Med. Biol. 32(6), 985-9.

Fritsch, A. and Hellmich, C. (2007), "'Universal' microstructural patterns in cortical and trabecular, extracellular and extravascular bone materials: micromechanics-based prediction of anisotropic elasticity," J. Theor. Biol. 244(4), 597-620.

Garcia, B., Cobbold, R., Foster, F., and McNeill, K. (1978), "Ultrasonic attenuation in bone," in IEEE Ultrasonics Symposium Proceedings, pp. 327-330.

Goss, S., Johnston, R., and Dunn, F. (1978), "Comprehensive compilation of empirical ultrasonic properties of mammalian tissues," J. Acoust. Soc Am. 64(2), 423-457.

Grimal, Q. and Naili, S. (2006), "A theoretical analysis in the time-domain of wave reflection on a bone plate," Journal of Sound and Vibration 298(1-2), 12-29.

Haiat, G., Lhemery, A., Renaud, F., Padilla, F., Laugier, P., and Naili, S. (2008a), "Velocity dispersion in trabecular bone: influence of multiple scattering and of absorption," J. Acoust. Soc. Am. 124(6), 4047-4058.

Haiat, G., Naili, S., Grimal, Q., Talmant, M., Desceliers, C., and Soize, C. (2009), "Influence of a gradient of material properties on ultrasonic wave propagation in cortical bone: application to axial transmission," J. Acoust. Soc. Am. 125(6), 4043-4052.

Haiat, G., Padilla, F., Barkmann, R., Dencks, S., Moser, U., Glüer, C., and Laugier, P. (2005), "Optimal prediction of bone mineral density with ultrasonic measurements in excised human femur." Calcif. Tissue Int. 77(3), 186-92.

Haiat, G., Padilla, F., Cleveland, R., and Laugier, P. (2006), "Effects of frequency dependent attenuation and dispersion on different speed of sound measurements on human intact femur." IEEE Trans. Ultrason. Ferroelectr. Freq. Control. 53(1), 39-51.

Haiat, G., Sasso, M., Naili, S., and Matsukawa, M. (2008b), "Ultrasonic velocity dispersion in bovine cortical bone: an experimental study," J. Acoust. Soc. Am. 124(3), 1811-1821. 
Han, S., Rho, J., Medige, J., and Ziv, I. (1996), "Ultrasound velocity and broadband attenuation over a wide range of bone mineral density," Osteoporos. Int. 6(4), 291-296.

Hans, D., Srivastav, S. K., Singal, C., Barkmann, R., Njeh, C. F., Kantorovich, E., Gluer, C. C., and Genant, H. K. (1999), "Does combining the results from multiple bone sites measured by a new quantitative ultrasound device improve discrimination of hip fracture?" J. Bone Miner. Res. 14(4), 644-651.

Lakes, R. S., Yoon, H. S., and Katz, J. L. (1986), "Ultrasonic wave propagation and attenuation in wet bone," J. Biomed. Eng. 8, 143-148.

Langton, C. M., Ali, A. V., Riggs, C. M., Evans, J. A., and Bonfield, W. (1990), "A contact method for the assessment of ultrasonic velocity and broadband attenuation in cortical and cancellous bone." Clin. Phys. Physiol. Meas. 11(3), 243-249.

Lees, S. and Klopholz, D. Z. (1992), "Sonic velocity and attenuation in wet compact cow femur for the frequency range 5 to 100 MHz." Ultrasound. Med. Biol. 18(3), 303-308.

Lehman, J. and Johnson, E. (1958), "Some factors influencing the temperature distribution in thighs exposed to ultrasound," Arch. Phys. Med. Rehab. 39, 347-356.

Macocco, K., Grimal, Q., Naili, S., and Soize, C. (2005), "Probabilistic modelling of an ultrasonic setup: calculation of the dispersion on wave speed measurements," Comptes Rendus Mecanique 333(7), 565-573.

Macocco, K., Grimal, Q., Naili, S., and Soize, C. (2006), "Elastoacoustic model with uncertain mechanical properties for ultrasonic wave velocity prediction; application to cortical bone evaluation," J. Acoust. Soc. Am. 119(2), 729-740.

Mayhew, P. M., Thomas, C. D., Clement, J. G., Loveridge, N., Beck, T. J., Bonfield, W., Burgoyne, C. J., and Reeve, J. (2005), "Relation between age, femoral neck cortical stability, and hip fracture risk," Lancet 366(9480), 129-135.

Moilanen, P., Talmant, M., Kilappa, V., Nicholson, P., Cheng, S., Timonen, J., and P., L. (2008), "Modeling the impact of soft tissue on axial transmission measurements of ultrasonic guided waves in human radius." J. Acoust. Soc. Am. 124(4), 2364-73.

Moser, F., Jacobs, L., and Qu, J. (1999), "Modeling elastic wave propagation in waveguides with the finite element method," NDT E int. 32.

Protopappas, V. C., Kourtis, I. C., Kourtis, L. C., Malizos, K. N., Massalas, C. V., and Fotiadis, D. I. (2007), "Three-dimensional finite element modeling of guided ultrasound wave propagation in intact and healing long bones," J. Acoust. Soc. Am. 121(6), 3907- 
3921.

Raum, K., Cleveland, R. O., Peyrin, F., and Laugier, P. (2006), "Derivation of elastic stiffness from site-matched mineral density and acoustic impedance maps," Phys. Med. Biol. 51(3), 747-758.

Raum, K., Leguerney, I., Chandelier, F., Bossy, E., Talmant, M., Saied, A., Peyrin, F., and Laugier, P. (2005), "Bone microstructure and elastic tissue properties are reflected in QUS axial transmission measurements," Ultrasound Med. Biol. 31(9), 1225-35.

Reilly, D. T. and Burnstein, A. H. (1974), "The mechanical properties of cortical bone," J. Bone Joint Surg. Am. 56, 1001-1022.

Rho, J. Y. (1996), "An ultrasonic method for measuring the elastic properties of human tibial cortical and cancellous bone," Ultrasonics 34(8), 777-783.

Rico, H. (1997), "The therapy of osteoporosis and the importance of cortical bone," Calcif. Tissue Int. 61, 431-432.

Royer, D. and Dieulesaint, E. (2000), Elastic Waves in Solids. I: Free and Guided Propagation (Springer).

Sasso, M., Haïat, G., Yamato, Y., Naili, S., and Matsukawa, M. (2007), "Frequency dependence of ultrasonic attenuation in bovine cortical bone: an in vitro study," Ultrasound Med. Biol. 33(12), 1933-1942.

Sasso, M., Haïat, G., Yamato, Y., Naili, S., and Matsukawa, M. (2008), "Dependence of ultrasonic attenuation on bone mass and microstructure in bovine cortical bone," J. Biomech. 41(2), 347-355.

Seeley, D. G., Browner, W. S., Nevitt, M. C., Genant, H. K., Scott, J. C., and Cummings, S. R. (1991), "Which fractures are associated with low appendicular bone mass in elderly women? The Study of Osteoporotic Fractures Research Group," Ann. Intern. Med. 115(11), 837-842.

Serpe, L. and Rho, J. (1996), "The nonlinear transition period of broadband ultrasound attenuation as bone density varies." Journal of Biomechanics 29(7), 963-966.

Stegman, M. R., Heaney, R. P., Travers-Gustafson, D., and Leist, J. (1995), "Cortical ultrasound velocity as an indicator of bone status," Osteoporos. Int. 5(5), 349-533.

Thomas, C. D., Feik, S. A., and Clement, J. G. (2005), "Regional variation of intracortical porosity in the midshaft of the human femur: age and sex differences," J. Anat. 206(2), $115-125$. 
Turner, C. H. (2002), "Biomechanics of bone: determinants of skeletal fragility and bone quality," Osteoporos. Int. 13(2), 97-104.

Wear, K. A. (2000), "The effects of frequency-dependant attenuation and dispersion on sound speed measurements: applications in human trabecular bone," IEEE Trans. Ultrason. Ferroelect. Freq. Contr. 47(1), 265-273.

Wear, K. A. (2001), "A numerical method to predict the effects of frequency-dependent attenuation and dispersion on speed of sound estimates in cancellous bone," J. Acoust. Soc. Am. 109(3), 1213-1218.

WHO Study Group (1994), "Assessment of fracture risk and its application to screening for postmenopausal osteoporosis," WHO Technical Report Series 843, World Health Organization, http://www.who.int/reproductive-health/publications/ageing.html (date last viewed $4 / 21 / 08)$.

Wismer, M. (2006), "Finite element analysis of broadband acoustic pulses through inhomogenous media with power law attenuation." J. Acoust. Soc Am. 120(6), 3493-502.

Zysset, P., Guo, X., Hoffler, C., Moore, K., and Goldstein, S. (1999), "Elastic modulus and hardness of cortical and trabecular bone lamellae measured by nanoindentation in the human femur," J. Biomech. 32(10), 1005-12. 
Table I. Mean, maximum and minimum values of the attenuation coefficient at $1 \mathrm{MHz}$ and of the corresponding viscosity of bone marrow and of soft tissues considered in the present study. These values are taken from Dussik and Fritch (1956); Goss et al. (1978); Lehman and Johnson (1958).

\begin{tabular}{ccr}
\hline \hline $\begin{array}{c}\text { Physical } \\
\text { property }\end{array}$ & $\begin{array}{c}\alpha_{L}(1 \mathrm{MHz}) \\
\left({\left.\mathrm{dB} . \mathrm{cm}^{-1}\right)}^{-1}\right.\end{array}$ & $\begin{array}{c}\eta_{L} \\
(\text { Pa.s })\end{array}$ \\
\hline Mean value (reference) & 1 & 1.97 \\
\hline Minimum value & 0 & 0 \\
\hline Maximum value & 2 & 3.94 \\
\hline \hline
\end{tabular}


Table II. Mean, maximum and minimum values of the four homogenized elastic constants and of mass density affecting the ultrasonic propagation in the framework of the 2-D model of Fig. 1.

\begin{tabular}{cccccc}
\hline \hline $\begin{array}{c}\text { Mechanical } \\
\text { quantity }\end{array}$ & $\begin{array}{c}C_{11} \\
(\mathrm{GPa})\end{array}$ & $\begin{array}{c}C_{13} \\
(\mathrm{GPa})\end{array}$ & $\begin{array}{c}C_{33} \\
(\mathrm{GPa})\end{array}$ & $\begin{array}{c}C_{55}=G_{L} \\
(\mathrm{GPa})\end{array}$ & $\begin{array}{c}\rho_{s} \\
\left(\mathrm{~g}_{\left.\mathrm{c} . \mathrm{cm}^{-3}\right)}\right.\end{array}$ \\
\hline $\begin{array}{c}\text { Mean } \\
\text { value } \\
\text { (reference) }\end{array}$ & 23.05 & 8.7 & 15.1 & 4.7 & 1.722 \\
\hline Minimum & 17.6 & 5.1 & 11.8 & 3.3 & 1.66 \\
\hline Maximum & 29.6 & 11.1 & 25.9 & 5.5 & 1.753 \\
\hline \hline
\end{tabular}


Table III. Mean, maximum and minimum values of the four homogenized elastic constants and of mass density affecting the ultrasonic propagation in the framework of the 2-D model of Fig. 1. These values are derived from Dong and Guo (2004).

\begin{tabular}{cccccccc}
\hline \hline $\begin{array}{c}\text { Mechanical } \\
\text { quantity }\end{array}$ & $\begin{array}{c}\alpha_{L, x} \\
\left(\mathrm{~dB} . \mathrm{cm}^{-1}\right)\end{array}$ & $\begin{array}{c}\alpha_{L, z} \\
\left(\mathrm{~dB} . \mathrm{cm}^{-1}\right)\end{array}$ & $\begin{array}{c}\alpha_{T} \\
\left(\mathrm{~dB} . \mathrm{cm}^{-1}\right)\end{array}$ & $\begin{array}{c}\eta_{11} \\
(\text { Pa.s })\end{array}$ & $\begin{array}{c}\eta_{33} \\
(\text { Pa.s })\end{array}$ & $\begin{array}{c}\eta_{55} \\
(\text { Pa.s })\end{array}$ & $\begin{array}{c}\eta_{13} \\
(\text { Pa.s })\end{array}$ \\
\hline $\begin{array}{c}\text { Mean } \\
\text { value } \\
\text { (reference) }\end{array}$ & 3.2 & 4.2 & 4 & 157 & 168 & 18.1 & 121.8 \\
\hline Minimum & 0.8 & 1.7 & 0 & 39.3 & 67.8 & 0 & 54 \\
\hline Maximum & 10.6 & 12.8 & 8 & 521 & 502 & 36.2 & 154 \\
\hline \hline
\end{tabular}


Table IV. Sensitivity of the FAS velocity to changes of the viscous properties of the soft tissues and of bone marrow for two values of the cortical thickness and homogeneous bone material properties. The computed FAS velocity is indicated for the minimal and maximal values of each variable corresponding to the realistic range of variation shown in Table II.

\begin{tabular}{|c|c|c|c|c|}
\hline $\begin{array}{c}\text { Material } \\
\text { property } S\end{array}$ & \multicolumn{2}{|c|}{$\begin{array}{c}\eta_{f} \text { in soft tissues } \\
(\text { Pa.s })\end{array}$} & \multicolumn{2}{|c|}{$\begin{array}{c}\eta_{f} \text { in bone marrow } \\
(\text { Pa.s })\end{array}$} \\
\hline $\begin{array}{c}\text { Cortical } \\
\text { thickness } h \\
(\mathrm{~mm})\end{array}$ & 0.6 & 4 & 0.6 & 4 \\
\hline $\begin{array}{c}\text { FAS velocity } \\
\text { for } S_{m} \\
\left(\mathrm{~m} . \mathrm{s}^{-1}\right)\end{array}$ & 3494 & 3731 & 3495 & 3731 \\
\hline $\begin{array}{c}\text { FAS velocity } \\
\text { for } S_{M} \\
\left(\mathrm{~m} . \mathrm{s}^{-1}\right)\end{array}$ & 3495 & 3732 & 3495 & 3731 \\
\hline
\end{tabular}


Table V. Sensitivity of the FAS velocity to changes of the viscoelastic properties of cortical bone for two values of the cortical thickness and homogeneous bone material properties. The computed FAS velocity is indicated for the minimal and maximal values of each variable corresponding to the realistic range of variation shown in Table II.

\begin{tabular}{|c|c|c|c|c|c|c|c|c|}
\hline $\begin{array}{c}\text { Material } \\
\text { property } S\end{array}$ & \multicolumn{2}{|c|}{$\begin{array}{c}\eta_{11} \\
(\text { Pa.s })\end{array}$} & \multicolumn{2}{|c|}{$\begin{array}{c}\eta_{13} \\
(\text { Pa.s })\end{array}$} & \multicolumn{2}{|c|}{$\begin{array}{c}\eta_{33} \\
(\text { Pa.s })\end{array}$} & \multicolumn{2}{|c|}{$\begin{array}{c}\eta_{55} \\
(\text { Pa.s })\end{array}$} \\
\hline $\begin{array}{l}\text { thickness } h \\
\text { (mm) }\end{array}$ & 0.6 & 4 & 0.6 & 4 & 0.6 & 4 & 0.6 & 4 \\
\hline $\begin{array}{c}\text { FAS velocity } \\
\text { for } S_{m} \\
\left(\mathrm{~m} \mathrm{~s}^{-1}\right)\end{array}$ & 3406 & 3654 & 3541 & 3738 & 3475 & 3732 & 3494 & 3734 \\
\hline $\begin{array}{c}\text { FAS velocity } \\
\text { for } S_{M} \\
\left(\mathrm{~m}^{-\mathrm{s}^{-1}}\right)\end{array}$ & 3747 & 3956 & 3468 & 3735 & 3545 & 3734 & 3494 & 3733 \\
\hline
\end{tabular}


Table VI. Contributing depths corresponding to spatial variations of different parameters. Results are shown for both types of gradient. The six last column corresponds to results obtained in Haiat et al. (2009). The values in bold correspond to non physical results (see text for explanations).

\begin{tabular}{|c|c|c|c|c|c|c|c|c|c|c|}
\hline $\begin{array}{c}\text { Material } \\
\text { property } S\end{array}$ & \multicolumn{2}{|c|}{$\begin{array}{c}\eta_{11} \\
(\text { Pa.s }) \\
\end{array}$} & \multicolumn{2}{|c|}{$\begin{array}{c}P(\%) \\
\text { (viscoelastic case) }\end{array}$} & \multicolumn{2}{|c|}{$\begin{array}{c}C_{11} \\
(\mathrm{GPa})\end{array}$} & \multicolumn{2}{|c|}{$\begin{array}{c}\rho_{s} \\
\left(\mathrm{~g} . \mathrm{cm}^{-3}\right)\end{array}$} & \multicolumn{2}{|c|}{$\begin{array}{c}P(\%) \\
\text { (elastic case) }\end{array}$} \\
\hline gradient & 1 & 2 & 1 & 2 & 1 & 2 & 1 & 2 & 1 & 2 \\
\hline \multicolumn{11}{|l|}{ Contributing } \\
\hline length (mm) & 1.68 & 1.55 & -0.04 & 1.8 & 0.59 & 0.66 & 1.03 & 1.04 & 0.46 & 0.63 \\
\hline
\end{tabular}




\section{LIST OF FIGURES}

1 Schematic representation of the simulation domain corresponding to a threelayer medium. The emitter and receivers are indicated by indents. . . . . . 44

2 Schematic representation of the two types of spatial variation considered for the material property $S$ corresponding the viscoelastic coefficient. The lines in the solid layer indicate the spatial dependence of $S$. The dotted line indicates homogeneous material properties corresponding the reference material properties. The variation of type 1 (respectively 2) shown in (a) (respectively (b)) corresponds to a constant value at the bone-soft tissues interface (respectively in the middle of the bone). . . . . . . . . . . . . . . . . .

3 Comparison between numerical (black solid lines) and analytical (gray dashed lines) results obtained for a plane wave propagation through an homogeneous transverse isotropic visoelastic medium. a) (b)): Attenuation coefficient of the longitudinal (transverse) wave mode, c) (d)): phase velocity of the longitudinal (transverse) wave mode. All velocities are plotted within the bandwidth of interest. . . . . . . . . . . . . . . . . . . . . . . . . .

4 Variation of the amplitude of the first arriving wave front as a function of the sensor number (increasing number corresponds to sensors farther to the emitter). The values of the components of the stiffness tensor are equal to the reference values indicated in Table I. The solid line indicates the results when no absorption was accounted for and the dashed line indicates the case where viscous losses are considered in both surrounding fluids as well as in the solid (reference values indicated in Table III are used for viscous and viscoelastic parameters).

5 Case of thick solid layer $h=4 \mathrm{~mm}$. Variation of the FAS velocity $\left(\mathrm{v}_{\mathrm{F}}\right)$ versus $\eta_{11}$ for homogeneous bone properties, the other material properties remaining equal to their reference value. The solid (respectively dashed) line indicate the results obtained using the finite element model (respectively the signal processing technique described in subsection ??). 
6 Case of thin solid layer $h=0.6 \mathrm{~mm}$. Variation of the FAS velocity $\left(\mathrm{v}_{\mathrm{F}}\right)$ versus $\eta_{11}$ (black solid line), $\eta_{13}$ (black dashed line) and $\eta_{33}$ (grey solid line) for homogeneous bone properties, the other material properties remaining equal to their reference value. . . . . . . . . . . . . . . . . . . . . . .

7 Variation of the FAS velocity $\left(\mathrm{v}_{\mathrm{F}}\right)$ versus porosity for homogeneous bone material properties. The black (respectively grey) solid line corresponds to the case of a thick (respectively thin) solid layer of $h=4 \mathrm{~mm}$ (respectively $h=0.6 \mathrm{~mm}$ ). . . . . . . . . . . . . . . . . . . . . . . 50

8 Case of thick solid layer $h=4 \mathrm{~mm}$. Variation of the FAS velocity $\left(\mathrm{v}_{\mathrm{F}}\right)$ versus $\delta_{11}$. The black lines correspond to the variation of type 1 (constant value of $\eta_{11}$ at $z=0$ ) and the grey lines correspond to the variation of type 2 (constant value of $\eta_{11}$ at $z=-0.5 h$ ). The continuous lines indicate the results obtained with the finite element model. The dashed lines correspond to the FAS velocity obtained with homogeneous spatially averaged viscoelastic properties (corresponding to the value at $z=-0.5 h$ ) obtained from Fig. 5.

9 Case of thick solid layer $h=0.6 \mathrm{~mm}$. Variation of $\mathrm{v}_{\mathrm{F}}$ versus (a) $\delta_{11}$, (b) $\delta_{33}$ and (c) $\delta_{13}$. The black lines correspond to the variation of type 1 (constant value of $\eta_{11}, \eta_{33}$ and $\eta_{13}$ at $\left.z=0\right)$ and the grey lines correspond to the variation of type 2 (constant value of $\eta_{11}, \eta_{33}$ and $\eta_{13}$ at $z=-0.5 h$ ). The continuous lines indicate the results obtained with the finite element model. The dashed lines correspond to the FAS velocity obtained with homogeneous spatially averaged viscoelastic properties (corresponding to the value at $z=-0.5 h$ ) obtained from Fig. 6. . . . . . . . . . . . . . . . . . . . . . . . . . 52 
10 Variation of $\mathrm{v}_{\mathrm{F}}$ versus porosity for (a) $h=0.6 \mathrm{~mm}$ and (b) $h=4 \mathrm{~mm}$. The black lines correspond to the variation of type 1 (constant value of $P$ at $z=0$ ) and the grey lines correspond to the variation of type 2 (constant value of $P$ at $z=-0.5 h)$. The solid lines indicate the results obtained when considering a simultaneous variation of mass density and of the stiffness and viscoelastic coefficients. The dashed lines indicate the results obtained when considering a simultaneous variation of mass density and of the stiffness coefficients only, bone being considered as an elastic material (see Haiat et al. (2009)). The thin dotted line shows the FAS velocity obtained when considering a constant homogeneous value of the porosity equal to its spatially averaged values, which corresponds to the value of the porosity at $z=-0.5 h . . . . . . . . .53$ 


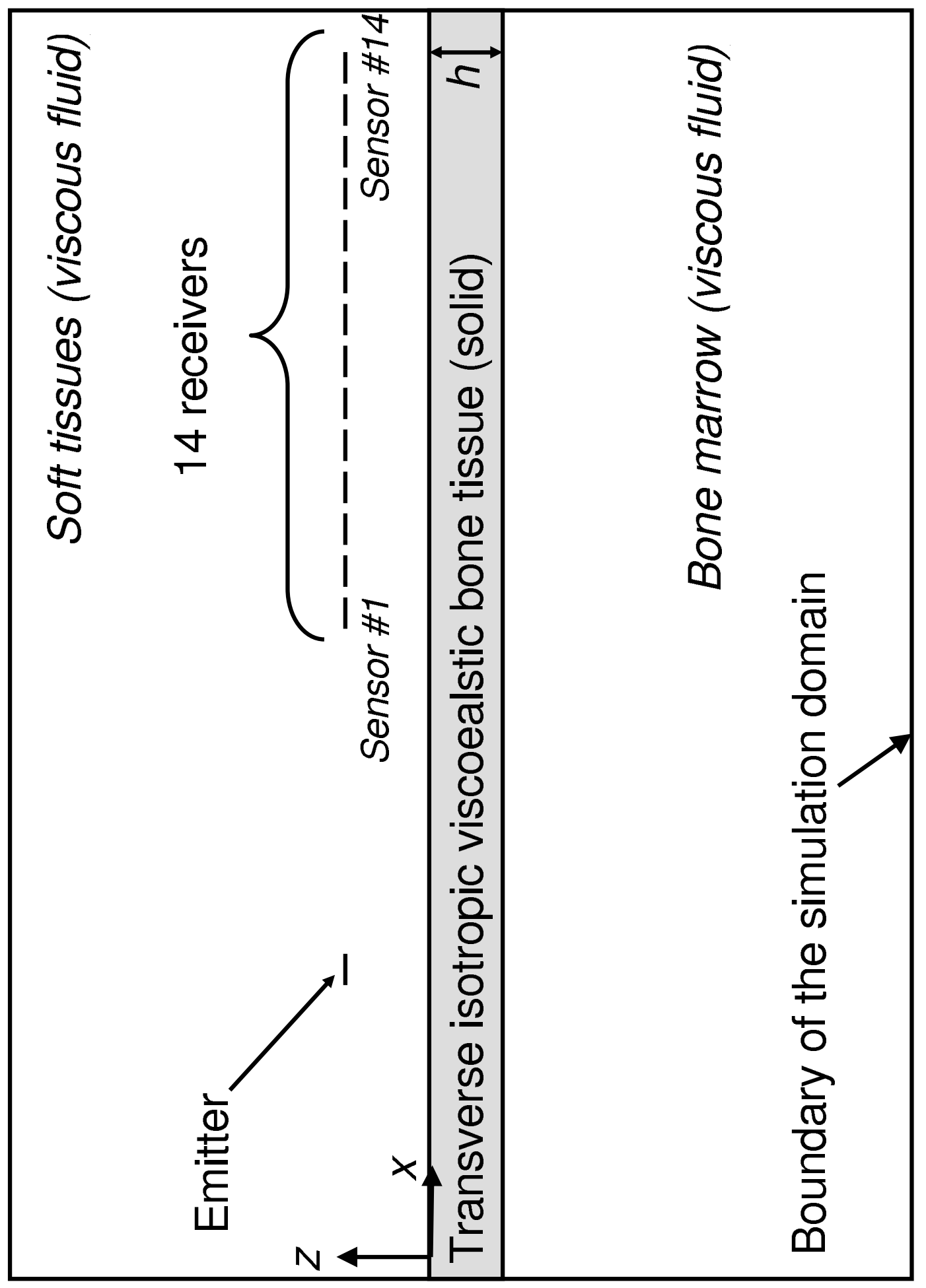

Figure 1. 


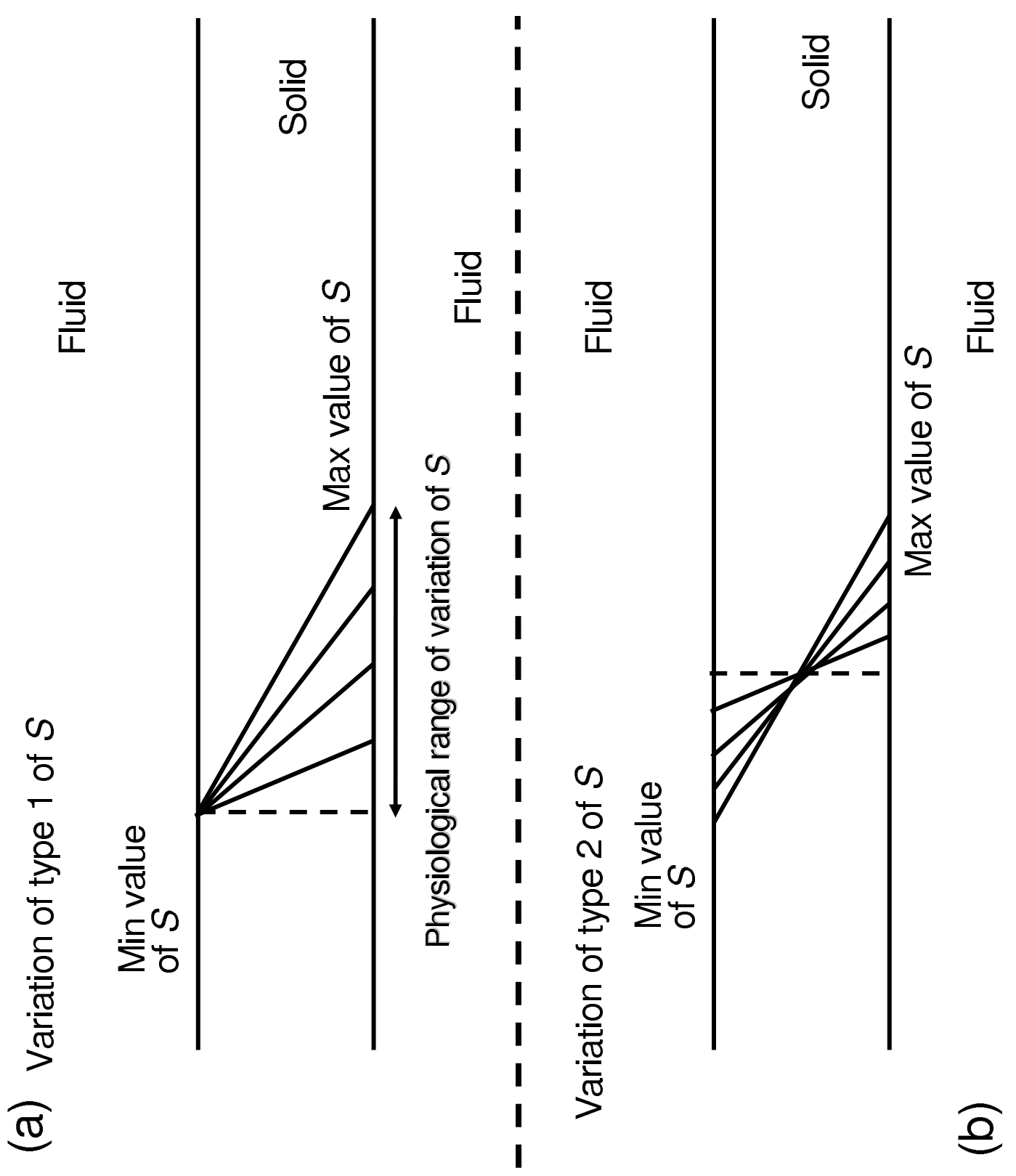

Figure 2. 

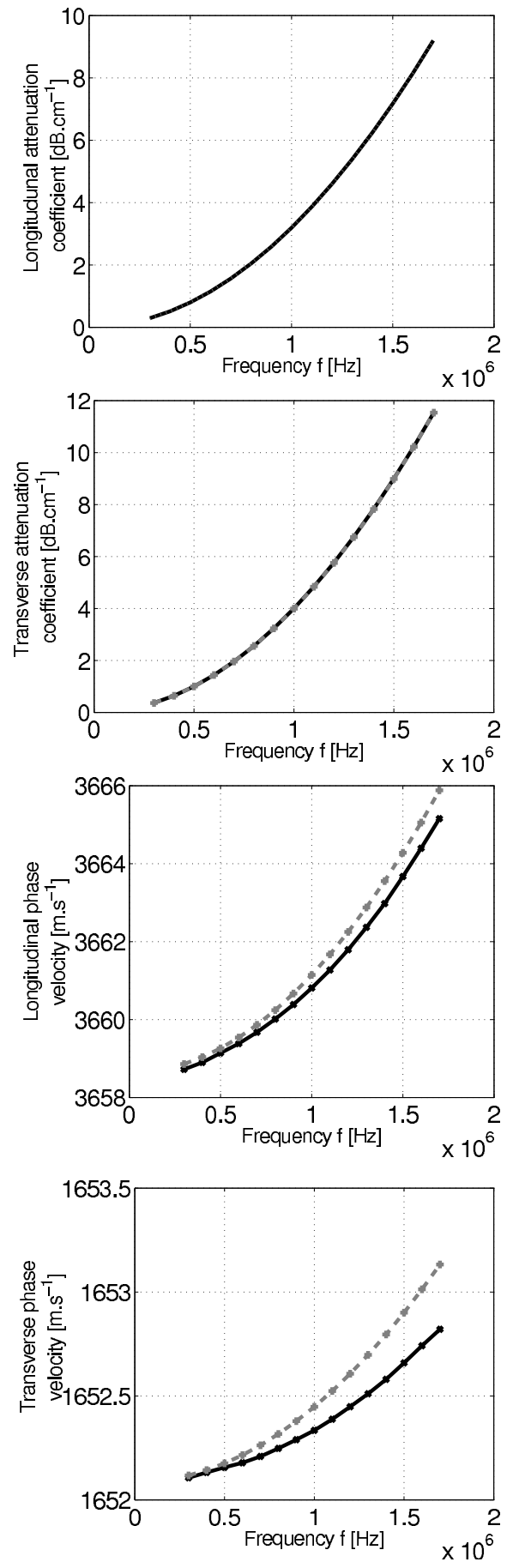

Figure 3. 


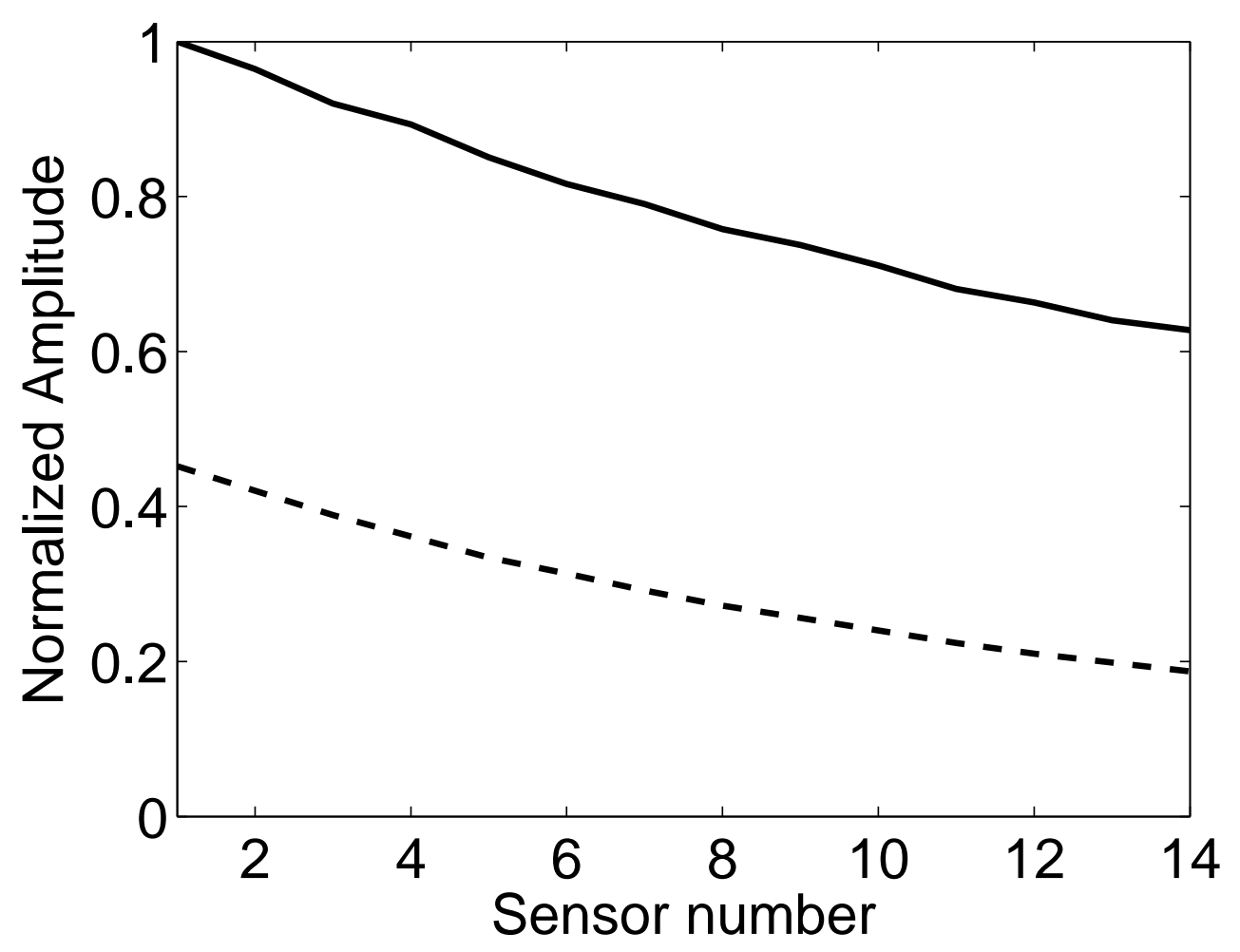

Figure 4. 


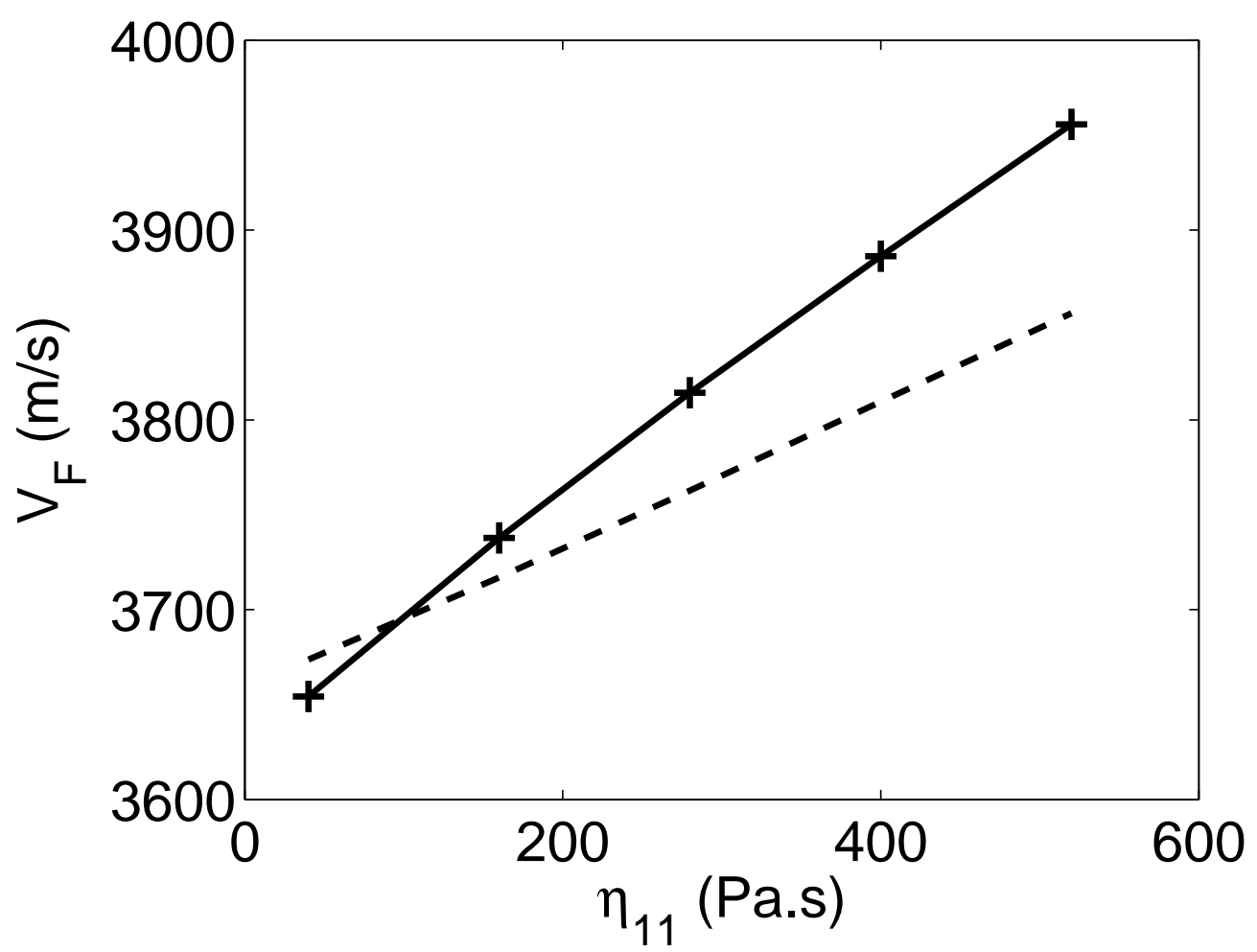

Figure 5. 


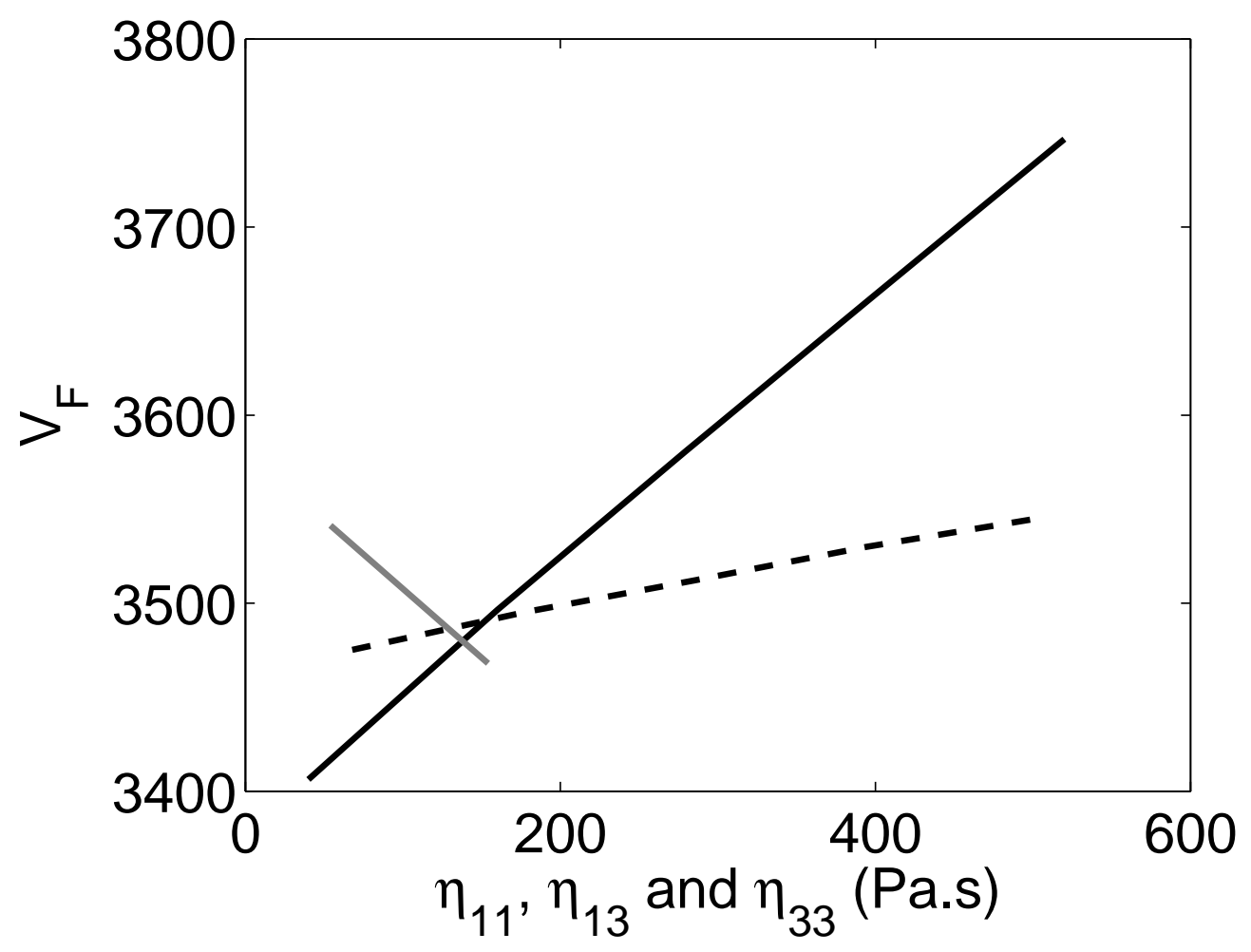

Figure 6. 


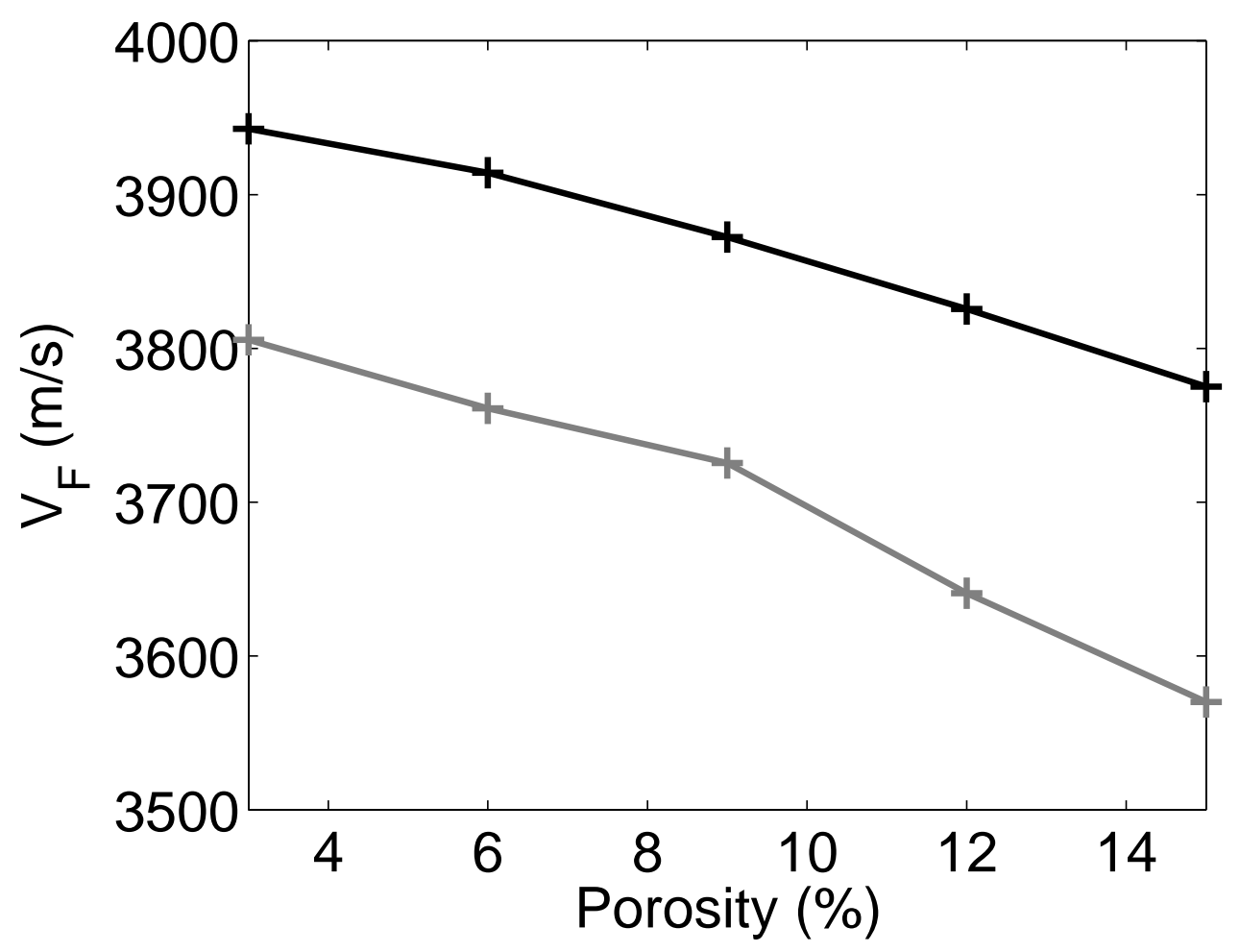

Figure 7. 


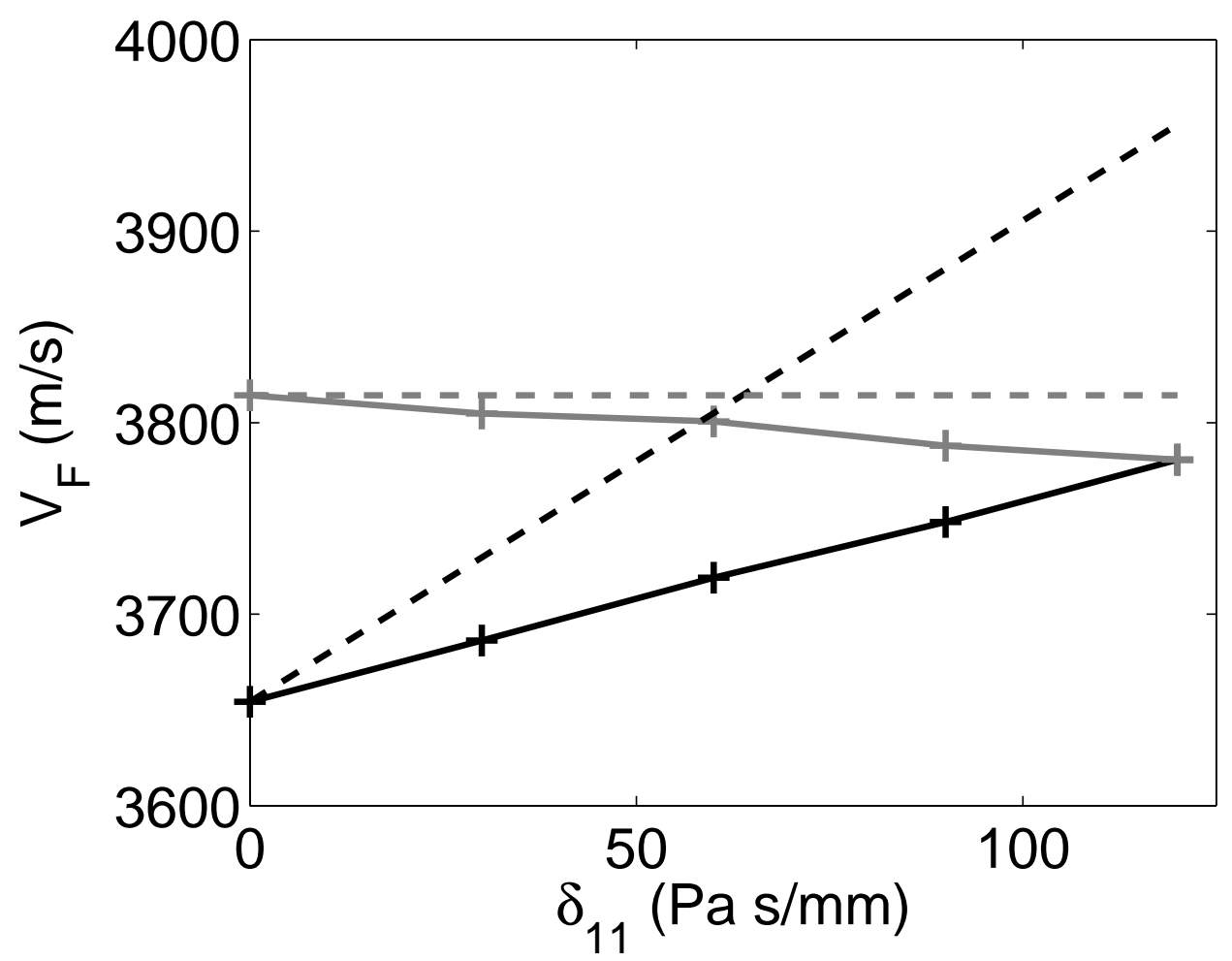

Figure 8. 

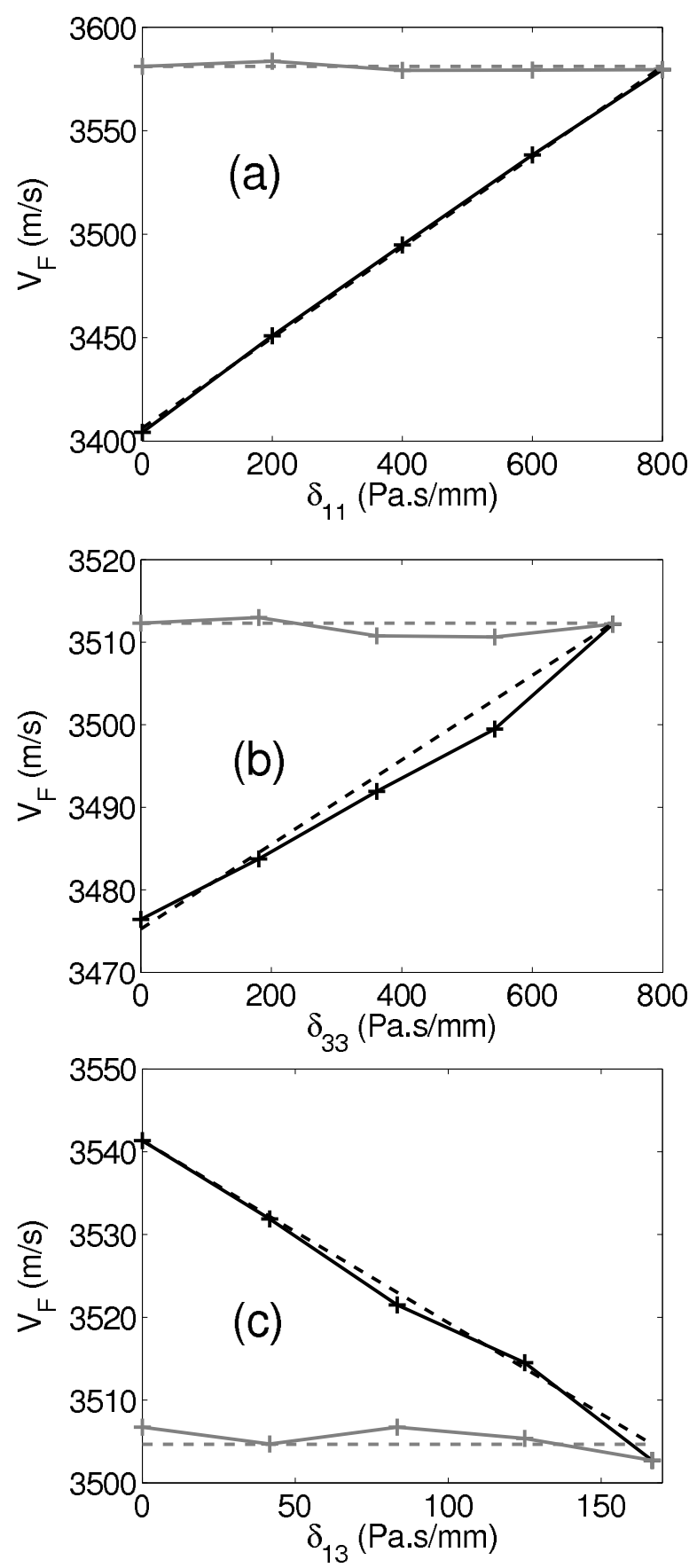

Figure 9. 

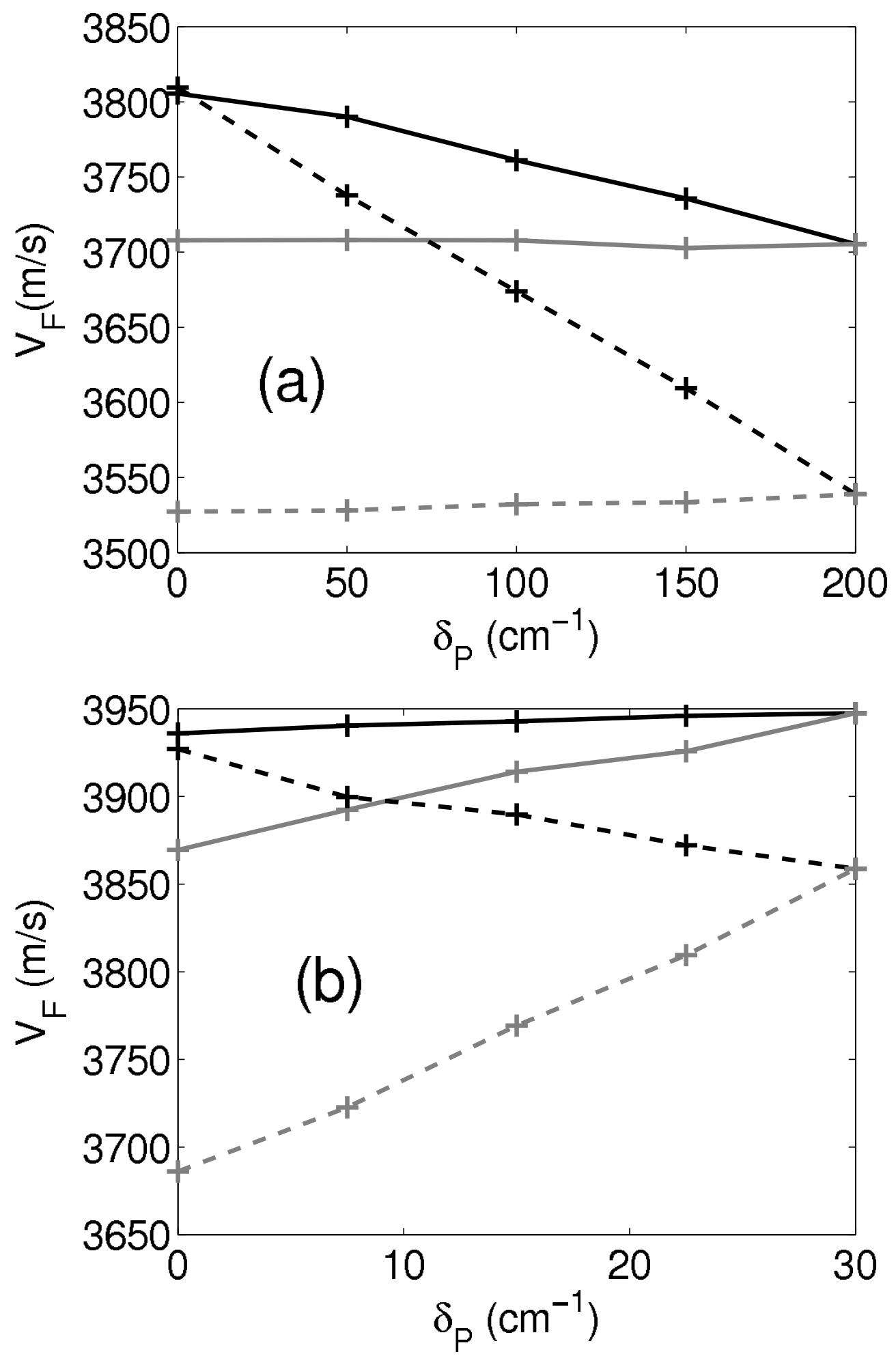

Figure 10. 